\title{
Inhibition of HIV-1 immune modulation by small molecules targeting viral Nef- host CD80 interface
}

Anusmrithi U. Sharma ${ }^{1,3}$, Shweta Sharma ${ }^{4,5}$, Gandhimathi Arumugam ${ }^{2}$, Archana Padmanabhan Nair ${ }^{3}$, Srinivas Ambala ${ }^{4,5}$, Gurunadham Munagala ${ }^{4,5}$, Kushalava Reddy Yempalla ${ }^{4,5}$, Akankshi Munjal2 ${ }^{2}$ Shreenidhi Rajkumar ${ }^{1}$, Neelagandan Kamariah ${ }^{1}$, Ashok R. Venkitaraman ${ }^{1,6}$, Ramanathan Sowdhamini², Taslimarif Saiyed ${ }^{3}$, Parvinder Pal Singh ${ }^{4,5 *}$, Ram A. Vishwakarma ${ }^{3,5 *}$, Satyajit Mayor ${ }^{2 * \#}$ and Anandi S. Karumbati ${ }^{1,3 *}$

${ }^{1}$ Centre for Chemical biology and Therapeutics, Institute of Stem Cell Science and Regenerative Medicine, GKVK, Bellary Road, Bangalore, India

${ }^{2}$ National Centre for Biological Sciences, GKVK Campus, Bellary Road, Bangalore, India

${ }^{3}$ Centre for Cellular and Molecular Platforms, GKVK Campus, Bellary Road, Bangalore, India.

${ }^{4}$ CSIR-Indian Institute of Integrative Medicine, Canal Road, Jammu-180001, India.

${ }^{5}$ Academy of Scientific and Innovative Research (AcSIR), Ghaziabad-201 002, India.

${ }^{6}$ The Cancer Science Institute of Singapore, Centre for Translational Medicine, National University of Singapore, Singapore 117599 \& Agency for Science, Technology and Research (A*STAR), 8A

Biomedical Grove, Singapore 138648.

* Co-corresponding authors

\# Further information and requests for resources and reagents should be directed to and will be fulfilled by the lead contact, Satyajit Mayor (mayor@ncbs.res.in)

\section{Abbreviations}

Nef (Negative Regulatory Factor)

MHC (Major Histocompatibility Complex)

CD80/86 (Cluster of Differentiation 80 and 86)

APC (Antigen Presenting Cells) 
AP (Aminopyrimidine)

PA (Phenoxyacetamide)

BC (Biaryl (heteroaryl) carbamate)

ART (antiretroviral therapy)

NNRTI (non-nucleoside inhibitors)

HAART (Highly Active Anti-Retroviral Therapy)

BnAb (Broadly neutralizing antibodies antibody)

LTNPs (Long-Term Non-Progressors)

MST (Micro-Scale Thermophoresis)

WST-1 (Water Soluble Tetrazolium-1)

SAXS (Small Angle X-ray scattering)

\section{Keywords}

immune modulation

HIV-1

Co stimulatory receptors- CD80/86

T-cell activation 


\section{Summary}

HIV-1 causes diverse immunomodulatory responses in the host, including the downregulation of co-stimulatory proteins CD80/86, mediated by HIV-1 protein Nef, blunting T-cell activation. Using a screening cascade of biochemical and cell-based assays, we identified potent small molecules representing three chemical scaffolds namely amino pyrimidine, phenoxy acetamide and bi-aryl heteroaryl carbamate which target the protein-protein interaction interface of CD80/86 and Nef with submicromolar potency. These molecules restore CD80/86 surface levels in HIV-1-Nef infected antigen presenting cells and T-cell activation. Nef-CD80 interface and small molecule binding sites were mapped by using computational docking and structural studies, followed by validation by mutational analysis. This analysis resulted in the identification of two key residues, K99 and R111, which were associated with downmodulation of CD80 surface levels by Nef and important for small molecule binding. Targeting these interacting residues disabled Nef-mediated down-modulation of CD80 surface levels, consequently restoring T-cell activation. Thus, we validate a new target, the Nef-CD80/86 protein-protein interaction interface, with a potential to develop new inhibitors to counteract the immunomodulatory consequences of HIV-1. 


\section{Introduction}

1 Over the past decade, there has been tremendous effort in finding newer therapies

2 for HIV/AIDS. Increased use of anti-retroviral drugs has been accompanied by the steady increase in HIV drug resistance and viremia that often result in

4 immunosuppression leading to morbidity ${ }^{3}$. Drug resistance is mainly transmitted at

5 the time of infection or acquired during previous treatment for instance in women

6 given anti-retroviral drugs to prevent mother-to-child transmission of HIV ${ }^{4}$. Other

7 treatment strategies like using anti-HIV-1 antibodies (bnAbs) are reported in combination with ART which have the capacity to impact on HIV-1-specific T cell

9 immune responses in infected humans but whether it controls the virus remains to be determined $^{5}$. In addition, with the vaccine trials failing to elicit broad plasma neutralization of primary virus isolates ${ }^{6}$, there is an urgent need to fast-track the transition to newer antiviral drug regimens which needs to be administered in multidrug combinations in order to combat the ever-evolving virus. Increasing drug resistance also emphasizes the requirement of new molecules that can target the host-viral interface with high specificity ${ }^{7,8}$.

To obtain targets for the next generation of HIV-1 therapy we explored the interaction

17 interface of the essential HIV-1 accessory protein, $\mathrm{Nef}^{9}$, with host proteins. Nef is a 27-35 kDa protein expressed during early phases of viral replication and helps maintain a constant state of infection by disrupting T-cell activation, thereby allowing evasion of the host immune system ${ }^{10,11,12}$. Evidence supporting a direct role for Nef in HIV disease comes from transgenic mouse models developed, in which a CD4derived promoter was used to express Nef in various tissues including thymus, kidney and lungs which progressed into AIDS-like phenotype, featuring CD4+ T-cell 24 loss, thymic involution, splenic atrophy and subsequent kidney and lung pathology ${ }^{13}$. 
25 This phenotype in many aspects' mimics human AIDS. Also in patient populations, fortuitous deletions in the nefgene in HIV infected patients of the Sydney Blood Bank Cohort remain essentially free of AIDS related symptoms ${ }^{14,15}$. In another study, the proportion of nefgene defects was found to be significantly higher in Long-Term Non-Progressors (LTNPs) compared to progressors ${ }^{16,17,18}$. These animal and patient studies suggests that Nef plays a pivotal role in pathogenesis and AIDS-like progression in HIV-infected individuals ${ }^{19}$.

Nef functions by re-engineering the levels of many surface proteins such as MHC-I, CD4, CD28, CXCR4, and CD3 in infected cells, and redirecting them to endosomes ${ }^{20,21}$. Nef interacts either directly or indirectly with multiple host partners and functions to increase the pathogenesis of the virus ${ }^{22,23}$. Our previous work has highlighted interactions of Nef with the host cell surface co-stimulatory proteins CD80 and CD86 24,25 . Down-regulation of CD80/86 is sufficient to cause impaired naïve Tcell stimulation in vitro and in vivo ${ }^{26}$. We have shown that by administering just the cytoplasmic tails of CD80 and CD86 in Nef-expressing cells, CD80/86 down modulation is prevented, making this host-viral interface amenable for developing new chemical biological tools and providing targets for therapeutic intervention.

42 The variety of functions carried out by Nef is based on its ability to interact with multiple cellular proteins. This is possibly due to its structure; it has many flexible regions, a feature unusual for cytoplasmic proteins. The unstructured regions of Nef may ease the allosteric adjustments required for interaction of Nef with different proteins. However, the flexible and unstructured features of Nef protein add substantial challenges to structurally characterize the full-length Nef protein and study their binding sites. 
In the current study, we show that Nef directly binds to cytoplasmic tail peptides of CD80 and CD86. We have identified small molecules, which can abrogate Nef interactions with CD80 and/or CD86. The compounds mainly belong to 3 scaffolds amino pyrimidine (AP), phenoxy acetamide (PA) and bi-aryl heteroaryl carbamate (BC) having nanomolar to micromolar inhibition potencies in vitro. Representative actives from these scaffolds were then validated in functional cell-based assays for reverting the down regulation of cell surface Nef-mediated co-stimulatory protein expression and re-establishing T-cell activation. These identified actives also reversed similar Nef-mediated effects after viral infections.

To further improve the efficacy of these leads, we used an in-silico approach to explore the binding mode of Nef with the co-stimulatory molecules. Full length Nef protein was modeled and potential binding sites for CD80 were identified. Based on these predictions a selected subset of CD80 interacting residues in Nef were mutated and the corresponding mutants were examined in assay platforms to confirm the model and its functional consequences. The understanding of the druggable pocket opens the scope for future lead optimization work. Altogether, we report a chemical strategy to inhibit Nef-mediated immunomodulatory functions, which prevents immune evasion of HIV-infected cells. While these molecules may be developed for future therapeutic intervention, at the current stage, they may be used as chemical biology tools to understand the role of host-pathogen interface in the form of Nef-CD80/86 interaction surface in HIV immune evasion. 
73 In our previous work, we have shown that cytoplasmic tail peptides from CD80 and

74 CD86 compete with the down modulation of surface CD80/86 by Nef protein in cell based assays ${ }^{25}$. For our assays we have used Nef F2 recombinant protein of Subtype-C origin (the alignment of the Nef sequence from this subtype with the commonly used subtype B is presented in Sup. Fig. S1a). To ascertain direct binding of Nef to the cytoplasmic tail peptide of the co-stimulatory receptors CD80 or CD86 in microscale thermophoresis (MST) assay ${ }^{27}$, we added 20-mer peptide of CD80 or CD86 (Fig. 1a, Sup. Fig. S1b) to fluorescently labelled Nef. A 16-point titration of peptides against Nef was carried out (see details in Star Methods). NefCD80 interaction showed a saturation curve with a kD of $27 \mu \mathrm{M}$ (Fig.1b). Nef-CD86 interaction showed a sigmoidal curve with $112 \mathrm{nM} \mathrm{kD}$, indicating a higher affinity interaction (Fig.1c). Having verified the direct binding of the CD80 and CD86 peptides with Nef in MST, we immobilized CD80/86 cytosolic peptides in a microtiter plate and examined the binding of Nef in an ELISA assay (Scheme, Sup. Fig. S1c). There was a 4-fold increase in the fluorescent signal due to binding of Nef to the 20mer CD80/86 cytosolic tail peptides compared to wells with an unrelated peptide (from CD74), or with no peptide controls (Fig. 1d). We used this ELISA format to screen and identify inhibitors of Nef-CD80 and Nef-CD86 interaction.

Small molecules were hand-picked for screening based on their drug-likeness and prior knowledge of potential protein-protein disruptors ${ }^{7}$. A threshold value was set to select actives with CD80 $\geq 30 \%$ and CD86 $\geq 20 \%$ cut-off for the Normalized Percent Inhibition. Based on this criterion, 33 actives belonging to nine scaffolds were identified from the screen (Fig.1e, f). Fig.1g depicts flow diagram of step-wise filtering process. After eliminating singleton scaffolds; we identified hits that belonged

97 primarily to three scaffolds namely amino pyrimidine (AP), phenoxy acetamide (PA) 

and bi-aryl heteroaryl carbamate (BC), which comprised of 20 compounds (Supp Table 1), and these were selected for resynthesis (Sup. Fig. S2). Compounds from all three scaffolds showed half maximal Inhibitory Concentration $\left(\mathrm{IC}_{50}\right)$ in nanomolar ranges (Fig. 1h; Supp Table 1). Two independent experimental dose response data correlated very well with an $\mathrm{R}^{2}$ value of 0.9 (Sup. Fig. S3).

In summary, the biochemical screen identified more interaction inhibitors of NefCD80 than Nef-CD86, which correlates well the MST binding isotherms indicating that the Nef-CD86 interaction is of higher affinity than the Nef-CD80 interaction. One representative compound from each scaffold with high potency against Nef-CD80 interaction was chosen for further cell-based assays.

\section{Small molecules block Nef-mediated internalization of cell surface CD80/CD86 receptors}

Before starting efficacy studies, we evaluated the cytotoxicity of the compounds (AP5, PA4 and BC5), as measured by WST-1 (Water Soluble Tetrazolium-1) assay. The cytotoxicity index at the highest concentration of $100 \mu \mathrm{M}$ for $24 \mathrm{~h}$ was around 17\% for AP5 and PA4 whereas BC5 was around 20\% (Sup. Fig. S4). The maximum concentration tested was $100 \mu \mathrm{M}$, and the concentrations chosen for the efficacy work were mostly non-toxic for the assay durations.

The cellular efficacy assay was developed based on observations from our previous work: the delivery of cytoplasmic CD80/86 tail peptides into the cell cytoplasm was able to compete with and abrogate Nef-mediated internalization of cell surface CD80/86 ${ }^{25}$. We expected the small molecules identified by the biochemical screen to behave in similar fashion to the cytosolic peptides. Surface levels of CD80/86 receptors in cells in culture with or without Nef protein were determined, and we 
observed a significant loss of surface levels of CD80 and CD86 in the presence of Nef in at least 3 types of Antigen Presenting Cells (APCs), including monocytes (Fig. 2a), consistent with our previous work ${ }^{25}$. The loss of $C D 80 / 86$ surface receptors was not observed with delivery of other non-specific proteins such as ovalbumin and $\beta$ lactoglobulin which confirms that the internalization of surface receptors CD80 and CD86 was indeed due to Nef (Sup. Fig. S5). We used RAJI, a B-lymphocyte cell line for our experiments since this cell line has high levels of CD80 and CD86, and chose to focus on targeting on the Nef-CD80 interface.

To test the effect of the compounds on Nef-mediated CD80 down modulation, cells were pre-treated with the AP5, PA4 and BC5 at two concentrations 10 and $100 \mu \mathrm{M}$ for 1 hour. Purified Nef protein was delivered into the cells and surface CD80 levels were analyzed by flow cytometry. AP5 inhibited Nef-mediated down modulation of CD80 at both $10 \mu \mathrm{M}\left({ }^{*} \mathrm{p} \leq 0.05\right)$ and $100 \mu \mathrm{M}\left({ }^{* *} \mathrm{p} \leq 0.01\right)$ thereby restoring CD80 levels. Compounds PA4 and BC5 also showed a significant restoration ( $\left.{ }^{*} p \leq 0.05\right)$ of surface CD80 levels only at $100 \mu \mathrm{M}$ (Fig. 2b).

We further validated the ability of these three compounds to reverse the effects of Nef in RAJl cells transduced with Nef-containing virus (YFP-tagged Nef) or Nefdeficient control virus (expressing only YFP). RAJl cells were pre-treated with AP5, PA4 and BC5 for 24 hours' prior-exposure to virus and further incubated with virus for $96 \mathrm{~h}$ and then surface levels of CD80 receptors were estimated by flow cytometry, there was $~ 50 \%$ loss of CD80 surface receptors in cells transduced with Nefcontaining virus as compared to control Nef-deficient virus infected cells. In cells pre-treated with compounds, all 3 compounds significantly reversed Nef-mediated internalization of CD80 receptors at $100 \mu \mathrm{M}$ (Fig. 2c). 


\section{Compound AP5 is a selective inhibitor for Nef-induced CD80 down modulation}

149 Since Nef also downregulates other cell surface molecules such as MHC-I and MHC-II in APCs and CD4 in T-cells, apart from CD80/86 during viral infection, we determined the effect of these compounds on the levels of $\mathrm{MHC}-\mathrm{I}^{28}$ in Neftransduced APCs. After compound treatment, we observed that PA4 and BC5 reversed Nef-mediated effect of MHC-I down modulation while AP5 did not alter Nefmediated down regulation of $\mathrm{MHC}-\mathrm{I}$ even at $100 \mu \mathrm{M}$ concentration (Fig. 2d). with CD80 and prevented the down modulation of CD86, the Nef-activated cascade that triggers MHC-I down modulation was reversed only by PA4 and BC5. This indicates a very specific role for AP5, and a more broad-spectrum role for PA4 and BC5.

\section{Restoration of T-cell activation in virus infected APCs by inhibitors}

162 The co-stimulatory receptors CD80/86 along with MHC-I are needed for T-cell activation. Previously it was shown that HIV-1 Nef-CD80/86 interaction impairs naïve

164 T-cell activation in in-vivo and in-vitro mouse systems ${ }^{24}$. We therefore ascertained if the small molecule inhibitors were able to reverse Nef-mediated T-cell inactivation. A co-culture assay system was adapted from a previously reported cell-based assay

167 for testing CD80 inhibitors ${ }^{29}$ (Fig. 3a). Briefly, a non-replicative retroviral vector with

168 Nef transgene (Sup Fig. 6 shows a comparison of the non-replicative retroviral vector with infectious HIV-1) was transduced in APCs to reduce CD80/86 levels at the surface. These APCs were then co-cultured with T-cells in the presence of anti-

171 CD3 antibody and functional T-Cell activation was assessed by measuring IL-2 
172 levels; relevant controls included T-cells alone, T-cells and B-cells in the absence of

173 anti- CD3 antibody (Fig. 3b).

174 We tested two different modes of addition of compounds: in the first mode, we pre-

175 treated APCs with 1, 10 and $100 \mu \mathrm{M}$ of compounds (AP5, PA4 and BC5) for 24h

176 then exposed the cells to Nef-carrying viral particles and assayed for IL-2 release.

177 We observed a dose dependent response with all 3 compounds AP5, PA4 and BC5

178 showing a 4-fold increase $\left({ }^{* *} p \leq 0.01\right)$ in IL-2 release at $100 \mu \mathrm{M}$ as compared to Nef

179 virus control. At lower concentrations, compounds AP5 and PA4 showed 2-fold

180 increase $\left({ }^{*} \mathrm{p} \leq 0.05\right)$ at $10 \mu \mathrm{M}$. PA4 did not show significant IL-2 release at $10 \mu \mathrm{M}$

181 (Fig. 3c), thus, indicating restoration of T-cell activation in a dose dependent

182 manner.

183 In the second mode, we added compounds at 1,10 and $100 \mu \mathrm{M}$ concentrations post

184 viral exposure for 96h. AP5 showed significant increase in IL-2 levels (3-fold) at all

185 concentrations when compared to Nef virus control $\left({ }^{* *} p \leq 0.01\right)$, while PA4 showed

1862 -fold increase ( $\left.{ }^{*} p \leq 0.05\right)$ in IL-2 levels at $10 \mu \mathrm{M}$ concentration and no changes in IL-

1872 levels were observed in BC5. Interestingly, there was a reduction in IL-2 release at

$188100 \mu \mathrm{M}$ dose in comparison to $1 \mu \mathrm{M}$ and $10 \mu \mathrm{M}$, perhaps due to the toxicity

189 associated with this dose alongside viral effects (Fig. 3d). Thus far, these data

190 shows that AP5 is a potent molecule both in vitro biochemical assays and in

191 restoring T-cell activation in an assay designed to assess the role of co-stimulation

192 dependent T-cell activation mediated by CD80/86.

194 Structural insights into Nef-CD80 interaction

195 To further understand the nature of inhibition of Nef-CD80 interaction by small

196 molecules such as AP5, we chose to analyze the structure of Nef-CD80-interaction 
197

198

199

200

201

202

203

204

205

206

207

208

209

210

211

212

213

214

215

216

217

218

219

220

pocket. This requires the characterization of the interaction surface between NefCD80 and an analysis of the ligand binding pocket for CD80. Despite repeated attempts we were unable to obtain crystals of full-length Nef that diffracted better than $4 \AA$, wherein the structure could be fully resolved. In the absence of a highresolution X-ray structure of the full-length Nef, we created a computational model of Nef using a multi-template modeling approach. The major structural information was acquired from the NMR structure PDB ID: 2NEF, as this structure has information for the highly flexible loop region of the core domain (55-66) which contains important interacting residues ${ }^{30}$, as well as from the crystal structure PDB ID: 3RBB which contains structural information of C-terminal folded core (residues 79-206). After modeling, the lowest energy state structure was obtained by energy minimization via SYBYL (Version 7.1) (Tripos Associates Inc.) and validated using PROCHECK. PROCHECK results for the model shows more than $95 \%$ of the residues are in allowed regions $(79.9 \%$ in the strictly allowed region and $17.2 \%$ in partially allowed region of the Ramachandran plot) which is better than the template structure $(62.3 \%$ in the strictly allowed region and $34.2 \%$ in partially allowed region of the Ramachandran plot).

The structure of full-length Nef can be divided into two parts: a flexible and structurally diverse $\mathrm{N}$-terminal region of about 70 residues followed by a wellconserved and folded core domain of about 120 amino acids. The core domain is the only part of the Nef protein which has a stable tertiary structure. It forms an $\alpha-\beta$ domain in which a central anti-parallel $\beta$-sheet of four strands $(\beta 1-\beta 4)$ is flanked by two long anti-parallel $\alpha$ helices ( $\alpha 4$ and $\alpha 5)$ and two short $\alpha$ helices ( $\alpha 1$ and $\alpha 5$ ). Residues 60-71 and 149-180 form flexible solvent exposed loops ${ }^{19}$ (Fig 4a). 
221 An independent verification of some aspects of the model was obtained from the predicted Small Angle X-ray Scattering (SAXS) envelope of soluble Nef protein. and $5 \mathrm{mg} / \mathrm{ml}$ (Sup. Fig. S7a). The Guinier plots at low angles appeared linear and confirmed good data quality with no indication of protein aggregation (inset Sup.

Fig. S7a). The derived $R_{g}$ values and the calculated maximum particle dimension ( $\left.D_{\max }\right)$ values were reported. The $R_{g}$ values extracted from the $P(r)$ function are in agreement with the $R_{g}$ values extracted from the Guinier region (Sup. Table 2). The estimated $R_{g}, D_{\max }$ and molecular mass of the full-length Nef suggest a concentration dependent increase in the $R_{g}, D_{\max }$ and molecular mass values observed with Nef addition. Visual inspection of the normalized Kratky plot reveals significant deviation from a bell-shaped profile which depict an inherent structural flexibility of Nef (Sup. Fig. S7b). The averaged solution shape calculated using the 1 $\mathrm{mg} / \mathrm{ml}$ scattering data clearly indicated that Nef is monomeric in solution. This solution model also revealed a two-domain architecture, a large domain that is well overlaid with the available 3D structure of folded C-terminal core (PDB ID: 3RBB) (Sup. Fig. S7c $)^{31,32}$. The small domain corresponds to the $\mathrm{N}$-terminal region (residues 1-78) that contain a long flexible loop (residues 24-68). While structural details of $\mathrm{N}$-terminal region are available from NMR studies of a peptide regions from residues $2-26^{33}$ and $2-57^{30}$, information about the relative orientation with $\mathrm{C}$-terminal core domain is missing. The SAXS data envelope along with the computational model (Fig. 4b) provides structural information about spatial arrangement of the Cterminal folded core and the flexible $\mathrm{N}$-terminal region of full-length Nef in solution, consistent with the computational predictions. 


\section{Identification of crucial residues involved in Nef-CD80 interaction surface}

247 We next utilized the computational prediction of full-length Nef structure and molecular docking studies with the cytoplasmic tail of CD80, to identify key residues at the interaction surface. The putative binding sites of cytoplasmic CD80 to full length Nef were mapped onto the template model utilizing SiteMap program for binding site prediction. In characterizing binding sites, SiteMap provided quantitative and graphical information in terms of site score and druggability score with properties such as hydrogen bond donor, acceptor, hydrophobic and hydrophilic regions in the predicted site. Docking studies of cytoplasmic tail region of CD80 with full length Nef revealed that CD80 may interact with the interface between the flexible $\mathrm{N}$-terminal and C-terminal core domain. Potential binding sites were predicted with a good site and druggability score (>0.5; Sup. Table 3).

The sites of interacting regions of Nef with other cellular proteins have been previously characterized. A polyproline motif (68-78aa) present on the core domain of Nef binds to the SH3 domain of Src kinases with high (nM to $\mu \mathrm{M}$ ) affinity ${ }^{34}$. Other than the polyproline motif within the core domain, a number of residues on the core domain are involved in multiple interactions, such as FPD $126-128$ with human thioesterase and $W_{61}$ and $L_{115}$ with $C D 4^{19}$. An acidic cluster $\left(E E E E_{65}\right)$ close to the core domain is required for interaction with PACS1 and controls MHC-I downregulation ${ }^{35,36}$. The unstructured regions of Nef also provide an extensive accessible surface that could be used to connect to other molecules. Since there is no prior information about the binding pattern of Nef with CD80 it was necessary to score each pose based on energy calculations. From the top ranked docked poses, the best complex with the lowest energy $(-246.30 \mathrm{kcal} / \mathrm{mol})$ was chosen as the model complex for Nef and CD80 interaction. In this predicted pose, CD80 cytoplasmic 
region (indicated in cyan) interacts with the Site- 1 and 2 residues in the core domain of Nef (indicated in blue) (Fig. 4c). Based on this pose, the interacting residues were mapped (left inset Fig. 4c). The side chains of site-1 residues $W_{61}, E_{68}, K_{99}$ and $\mathrm{R}_{111}$ are in favourable position to interact with the $\mathrm{N}$-terminus of the CD80 cytoplasmic tail. It should be noted that the residues $\mathrm{K}_{99}$ and $\mathrm{R}_{111}$ potentially make polar contact with CD80 backbone carbonyl oxygen of $\mathrm{F}_{4}$ and side chain hydroxyl group of $Y_{2}$, respectively. In addition, the C-terminus of the CD80 cytoplasmic tail potentially interact with $E_{160}$ and $D_{180}$ residues. The side of $E_{160}$ potentially makes salt bridge interaction with $\mathrm{R}_{16}$ and $\mathrm{R}_{23}$ of $\mathrm{CD} 80$ (right inset Fig. 4c,). Based on our insilico predictions we chose four residues that include, 3 from Site-1 ( $\mathrm{W}_{61}, \mathrm{~K}_{99}$ and $\left.R_{111}\right)$ and one from Site-2 $\left(E_{160}\right)$ for further analysis.

\section{Functional validation of predicted residues mediating Nef-CD80 interaction}

Single site mutant of Nef such as Nef ${ }^{\mathrm{W} 61 \mathrm{~A}}$, Nef ${ }^{\mathrm{K} 99 \mathrm{~A}}$, Nef ${ }^{\mathrm{R} 111 \mathrm{~A}}$ and Nef ${ }^{\mathrm{E} 160 \mathrm{~A}}$ were designed and purified (Sup. Fig. 8a \& b). These mutants were tested for their affinity for CD80 peptide in the ELISA assay (Fig. 5a). Two mutants Nef ${ }^{\mathrm{K} 99 \mathrm{~A}}$ and Nef ${ }^{\mathrm{R} 111 \mathrm{~A}}$ showed a loss of affinity to the CD80 peptide, whereas, the Nef ${ }^{\mathrm{W} 61 \mathrm{~A}}$ and Nef ${ }^{\mathrm{E} 160 \mathrm{~A}}$ exhibited an affinity comparable to full length Nef ${ }^{W T}$. These mutants were also assessed for their ability to affect CD80 surface levels after delivery into APCs. Indeed, the two mutants $\mathrm{Ne}^{\mathrm{K} 99 \mathrm{~A}}$ and Nef ${ }^{\mathrm{R} 111 \mathrm{~A}}$ did not show any reduction in CD80 levels, while Nef ${ }^{\mathrm{W} 61 \mathrm{~A}}$ showed slightly lesser reduction in CD80 receptors and Nef ${ }^{\mathrm{E} 160 \mathrm{~A}}$ behaved similar to $\mathrm{Nef}^{\mathrm{WT}}$ (Fig. 5 b). Consistent with a key role for the $\mathrm{K}_{99}$ and $\mathrm{R}_{111}$ in Nef-CD80 interactions, when transduced into APCs, Nef ${ }^{\mathrm{K} 99 \mathrm{~A}}$ and Nef ${ }^{\mathrm{R} 111 \mathrm{~A}}$ mutants did not affect IL-2 release (Fig. 5c) thereby showing that T-cell activation is not compromised by these mutant Nef variants. Surprisingly, while Nef ${ }^{\mathrm{W} 61 \mathrm{~A}}$ down 
modulated CD80 significantly, it did not result in a loss of T-cell activation. Since Tcell activation assay requires a minimum of $4-5 \mathrm{~h}$ we reasoned that the Nef ${ }^{\mathrm{W} 61 \mathrm{~A}}$ protein delivered into the APCs may be less stable than the other isoforms for the 5 $\mathrm{h}$ required for this assay. Indeed, western blot analysis of the protein at $2 \mathrm{~h}$ versus 5 $\mathrm{h}$ in cell lysates shows that the level of Nef ${ }^{\mathrm{W6} 1 \mathrm{~A}}$ protein was drastically decreased after $5 \mathrm{~h}$, while the levels of the other Nef protein variants remained substantial (Sup

Fig. 8c). The other Nef ${ }^{E 160 A}$ mutant exhibited similar reduction of IL-2 release as Nef ${ }^{\mathrm{WT}}$ consistent with its ability to bind CD80 peptides as well as down modulate CD80 at the APC surface (Fig. 5 b-d).

The two mutants $\mathrm{Ne}^{\mathrm{W} 61 \mathrm{~A}}$ and $\mathrm{Nef}^{\mathrm{R} 111 \mathrm{~A}}$ also did not down regulate MHC-I receptors as much as $\mathrm{Nef}^{\mathrm{WT}}$; whereas $\mathrm{Nef}^{\mathrm{K} 99 \mathrm{~A}}$ and $\mathrm{Ne}^{\mathrm{E} 160 \mathrm{~A}}$ exhibited a similar reduction in MHC-I levels as Nef ${ }^{\mathrm{WT}}$, as assessed by surface MHC-I antibody staining (Fig. $\mathbf{5 d}$ ). $W_{61}$ residue ( $W_{57}$ in Subtype $B$ ) has been previously reported to be important in CD4 down regulation and $R_{111}$ residue $\left(R_{106}\right.$ in subtype $\left.B\right)$ is located in the oligomerization domain of Nef. Many residues of Nef have been identified that promote interaction with MHC-I, including $\mathrm{W}_{57}$ and $\mathrm{R}_{106}$ in subtype-B, NL4-3 strain, but their mutation did not hinder MHC-I down regulation ${ }^{37}$. However, $\mathrm{W}_{61}$ and $\mathrm{R}_{111}$ residues in subtype $\mathrm{C}$, appear potentially important for Nef interaction with MHC-I, indicating subtle differences in the modulation of host proteins by different Nef variants. Nevertheless, these functional studies provide strong support to the predicted binding mode of CD80 peptide with Nef via residues $K_{99}$ and $R_{111}$ in Site 1 (Fig. 4c). Mutation in these residues leads to loss of binding capacity, resulting in the inability to down-modulate CD80 thereby restoring T-cell activation function of the transduced APCs. 
321 Considering the predictive potential of the computational model of Nef-CD80

322 interaction surface, the most potent binding inhibitor molecule, AP5 was docked with

323 Nef protein to capture its binding pattern and important residues involved in

324 interaction. AP5 fits nicely into the hydrophobic cavity formed by the residues $\mathrm{W}_{61}$,

$325 V_{71}, L_{115}$ and $W_{118}$, which are part of the $N$-terminal loop region and the $\alpha 4$ helix of core domain (Fig. 6a and inset). The aromatic residues $W_{61}$ and $W_{118}$ mainly have $\pi$ $-\pi$ stacking interaction with aromatic ring B in AP5. In addition to hydrophobic interactions, the side chain of $S_{50}$ and backbone of $E_{68}$ form hydrogen bond interaction with amine group of the ligand. The side chain of K9g from $\alpha 3$ helix of core domain, interacts with the $\mathrm{CF}_{3}$ (triflouro methyl) group. Moreover, AP5 binding site overlaps with Site-1 of Nef-CD80 binding pocket, and the docking results showed that the Nef-CD80 and Nef-AP5 binding sites are overlapping with two important common residues such as $\mathrm{W}_{61}$ and $\mathrm{K}_{99}$.

334 Consistent with these predictions, at $10 \mu \mathrm{M}$ AP5 was neither able to inhibit the interaction between $\mathrm{CD} 80$ peptide and $\mathrm{Nef}^{\mathrm{W} 61 \mathrm{~A}}$ mutant nor further reduce the residual interaction of Nef ${ }^{\mathrm{K} 99 \mathrm{~A}}$ and Nef ${ }^{\mathrm{R} 111 \mathrm{~A}}$ mutants in vitro (Fig. 6b). However, AP5 was able to displace both $\mathrm{Nef}^{\mathrm{WT}}$ and Nef ${ }^{\mathrm{E} 160 \mathrm{~A}}$ from CD80 peptides adsorbed on the ELISA plate. Thus, $\mathbf{W}_{61}$ is an important residue for AP5 binding to Nef. Furthermore, in agreement with the predictions, AP5 treatment did not result in any change in surface levels of CD80 in APCs transduced with Nef ${ }^{\mathrm{W} 61 \mathrm{~A}}$, Nef ${ }^{\mathrm{K} 99 \mathrm{~A}}$ and Nef $\mathrm{f}^{\mathrm{R} 111 \mathrm{~A}}$ mutant proteins (Fig. 6c). The levels of IL-2 release in all three mutants Nef ${ }^{\mathrm{W} 61 \mathrm{~A}}$, $\mathrm{Ne}^{\mathrm{K} 99 \mathrm{~A}}$ and $\mathrm{Ne} \mathrm{f}^{\mathrm{R} 111 \mathrm{~A}}$ also remained unchanged, with the Nef ${ }^{\mathrm{W} 61 \mathrm{~A}}$ mutant mimicking

343 the inhibition observed with wild type Nef. AP5 restored IL-2 release in Nef ${ }^{\mathrm{E} 160 \mathrm{~A}}$

344 treated cells, comparable to $\mathrm{Nef}^{\mathrm{WT}}$ (Fig. 6d), consistent with the inability of Nef ${ }^{\mathrm{E} 160 \mathrm{~A}}$

345 to affect neither CD80 nor AP5 binding, thereby serving as a negative control. These 
results predict and functionally validate the residues in the Nef protein that are important for AP5 binding.

\section{Hit refinement of AP series}

350 We chose AP5 as our starting point, given its nanomolar and micromolar potencies in biochemical and cell-based assays respectively as well as specificity to CD80. An initial hit refinement of AP5 was conducted to understand the preliminary structureactivity relationships (SAR). New analogs were synthesized in two series by modifying the rings $\mathrm{B}$ and $\mathrm{C}$ with un/substituted aryl (heteroaryl) moieties, and without $-\mathrm{CF}_{3}$ group at the $6^{\text {th }}$ position (Fig. 7a). In series-1, four analogs AP(S1-S4) were prepared (Sup. Fig. S2; Scheme-3), where phenyl group (ring B) was placed at the $4^{\text {th }}$ position of ring $A$ and varying the substitution pattern at $5^{\text {th }}$ position (ring $B$ ). In series-2, six analogs AP(S5-S10) were prepared by modifying both $4^{\text {th }}$ and $5^{\text {th }}$ positions (Sup. Fig. S2; Scheme-4). Apart from these, another analog AP-S11 (Sup. Fig. S2; Scheme-2) was also synthesized where - $\mathrm{CF}_{3}$ was maintained at the $6^{\text {th }}$ position and substituted aryl rings at $4^{\text {th }}$ and $5^{\text {th }}$ positions.

All the synthesized molecules were evaluated for their effect on Nef-CD80/CD86 inhibition by ELISA. The analogs AP(S1-S10) without $-\mathrm{CF}_{3}$ group at $6^{\text {th }}$ position of ring A didn't show any activity (Fig. 7b), however, the analog AP-S11 with $\mathrm{CF}_{3}$ at $6^{\text {th }}$ position of ring A showed the activity. These results disclosed that presence of $\mathrm{CF}_{3}$ group at $6^{\text {th }}$ position of ring $A$ important for the activity (Fig. 7c). Moreover, docking studies revealed that $\mathrm{CF}_{3}$-group showed hydrophobic interactions with non-polar residues such as $W_{61}, L_{91}, l_{114}$ and $L_{115}$ while in vitro and in vivo experiments described above, suggested that these interactions are important for at least for the

370 inhibition of the down modulation capacity of Nef. These studies underline the 
importance of the $-\mathrm{CF}_{3}$ group at $6^{\text {th }}$ position of ring A (Fig.7a-c). In conclusion, lead compounds with potency in nanomolar range across the cell-based assay along with acceptable solubility, permeability and pharmacokinetics parameters necessary for further drug development and chemical perturbation of the Nef-CD80 interface, have been developed.

\section{Discussion}

HIV-Nef plays an important role in the pathogenesis of HIV infections and understanding its many functions in modifying the host cell surface has served as a focal point of HIV research ${ }^{38,39}$. In earlier work we had determined that the presence of wild type Nef in virus infected cells, promotes the loss of the co-stimulatory proteins CD80/86 from the infected APC surface resulting in a loss of naïve T-cell

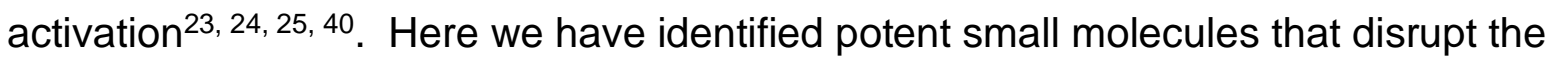
interaction of Nef with CD80 co-stimulatory receptors, and restore T-Cell activation potential of virus-infected APCs. The three lead structures identified: AP5, BC5 and PA4 belonged to three diverse scaffolds. While PA4 and BC5 are able to inhibit both Nef mediated CD80 as well as MHC1 down-regulation, possibly indicating different interaction points in Nef or counteracting Nef at more than one proteinprotein interface. PA4 and BC5 molecules are leads to explore for molecular interaction promiscuity and investigating some of the multiple interactions of Nef.

Since AP5 selectively inhibits Nef-mediated CD80 down-regulation, we chose to pursue its detailed characterization in this study,

394 To gain an insight into the interactions of Nef with CD80, computational approaches followed by experimental validation were used to identify possible binding sites on 
Nef for both CD80 peptide and the small molecule inhibitor AP5. Due to the lack of crystals with suitable diffraction properties to provide high-resolution structures, and limited experimental information on full length Nef structure, possibly due to its inherent flexibility we adopted a computational strategy. Full length Nef was modeled by a multi-template computational approach, and their spatial conformation was validated using the constraints obtained from SAXS experiments. The interaction site for CD80 was obtained by docking of CD80 cytoplasmic tail with full length Nef model, and a number of possible binding sites for its already known protein-protein interaction sites were identified. These predictions provide key insights that could be correlated with the experimental results, identifying key residues that are involved in Nef-CD80 interaction. A caveat to be noted is that there is a limitation in finding the best biologically relevant orientation of CD80 since the docking was restricted to only the cytoplasmic tail peptide of CD80 which does not impose spatial conformation of the full length CD80 embedded in the membrane. The free cytoplasmic CD80 region fits in the energetically favorable orientation, given its steric constraints, providing

411 verifiable insights from mutation studies. In parallel the AP5 docking results confirms 412 that $W_{61}$ and $K_{99}$ residues of Nef contributes to the interaction interface with this lead molecule. Based on the Nef-CD80 and Nef-AP5 docking results, hotspot residues such as $W_{61}, K_{99}$ and $R_{111}$ were mutated. This revealed that the $K_{99}$ and $R_{111}$ residues are crucial for CD80 binding and additionally, W61 plays an important role in 416 AP5 binding. Since the binding sites for CD80 and AP5 are overlapping, our study 417 provides a plausible view of the inhibitory mechanism, where AP5 interactions with 418 Nef would prevent its ability to associate with CD80, since they compete for the 419 same site. Ongoing efforts are aimed at improving the inhibitors with - $\mathrm{CF}_{3}$ group in 420 AP5 series and further validating these hits in in vivo studies. 
422 Our results also indicate that Nef interaction with the co-stimulatory receptors

423 CD80/86 cytoplasmic tails are distinct from reported Nef $-\mathrm{MHC}-\mathrm{I}$ interactions. Nef

424 interacts with $\mathrm{MHC}-\mathrm{I}$ cytoplasmic via $\mathrm{E}_{62-65}$ and $\mathrm{P}_{78}$ residues $^{41}$, although subtype $\mathrm{C}$

425 as shown here may utilize $\mathrm{W}_{61}$ and $\mathrm{R}_{111}$. While Nef-MHC-1 interactions are

426 important, Chaudhry et $a^{R 4}$ showed that the kinetics of Nef down-modulation of MHC

427 molecules is slow as compared to the loss of co-stimulatory CD80/86 function. We

have shown that the interaction of Nef with the co-stimulatory CD80/86 is likely to be

critical for inhibiting the priming of the immune system towards naïve infections, and

430 hence may be critical for immune evasion strategy of the virus ${ }^{42,43}$. The chemical

431 tools developed here will allow such an interrogation in suitable animal models.

432

433 Once developed into drug-like chemicals, the leads we have identified from this

434 study would have significant impact in at least two scenarios where macrophages

435 play an important role for HIV-1 pathogenesis. One important application is in maternal-fetal transmission and other in cases of early infection or persistent

437 infection resulting from viral mutations. $90 \%$ of HIV infection in children is through Macrophage-tropic Maternal-fetal Transmission ${ }^{44}$. Nef plays a major role in vertical transmission of Macrophage-tropic HIV-1 in mother to child, where the motifs for

440 receptor modulation were conserved in mother-to-infant NEF sequences. The second scenario is in early stages of infection when viral load is low, the role of Nefmediated CD80 and CD86 down modulation from the antigen-presenting cell surface could delay the onset of T-cell responses to provide the virus with a time window sufficient for expansion. There is evidence that T-cells need co-stimulatory

445 molecules for optimal killing of target cells, thus even in cases of established 
infection the removal of CD80 and CD86 from infected cell surfaces could reduce the

447 efficiency of T-cell responses ${ }^{45}$. If this is the case, blockade of this function by smallmolecule inhibitors of Nef-CD80/CD86-cytosolic tail interactions, could enhance effectors antiviral immunity and delay the onset of disease in both pathophysiological contexts.

452 Altogether, targeting this protein-protein interaction interface represents a promising new therapeutic approach to bring forth a first in class set of inhibitors to foreshorten the infection burden in HIV-1. The information gained from this integrated approach of both computational and experimental study have set the foundation for further ongoing efforts in synthesizing the next series of more drug-like inhibitors. We have demonstrated a promising chemical starting point for building chemical tools and drugs that can interfere with immunomodulatory consequences of HIV-1.

\section{Significance}

461 Our study aims to develop small molecule inhibitors that disrupt the interaction interface between HIV-1 viral protein Nef and host CD80 / CD86 in Antigen Presenting Cells (APC). The disruption of this interaction will makes infected APCs more visible to the immune system, increasing cytotoxic lymphocyte activity on these HIV-infected cells, potentially leading to viral clearance from macrophage reservoirs. Here we identify and structurally characterize small molecule inhibitors that indeed disrupt the protein-protein interaction interface of Nef-CD80 and restore the T-cell activation capacity of infected APCs. These chemical tools serve as excellent starting points that may be used to interrogate the role of Nef in HIV immune evasion and contribute to first-in-class drugs for mitigating this resurgent disease 
471 Acknowledgments

472 This work was funded by the BIRAC-CRS grant (BT/CRS0045/CRS -02/12), J.C.

473 Bose Fellowship from the Department of Science and Technology to SM,

474 Government of India, IIIM funds CSIR Research grants GAP-2116 and HCP-0001.

475 Center for Chemical Biology \& Therapeutics fund (sanction order no.

476 BT/PR7222/MED/31/1901/2012, dated 11.01.2013), and support from the

477 Department of Atomic Energy (Government of India) under Project No. RTI 4006 to NCBS. We thank Drs. Satyajit Rath, Ramaswamy Subramanian and Shahid Jameel for helpful discussions. We also thank M. Hurakadli, J. Subbarao, P.Kumar for their technical assistance. The facilities provided by the Bangalore Life Science (BLiSc): Biosafety-I \& II, CIFF, Instrumentation, X-ray facilities have greatly supported this work.

\section{Author contributions}

SM, ASK, TS, PPS and RAV contributed to project initiation and funding.

SM and ASK contributed to the study design of biochemical screen and cell-based assays. PPS and RAV contributed to the medicinal chemistry study design. ARV contributed to the structural biology study design and supported this work at the CCBT. AUS, APN and AM contributed to compound screening and cell-based experimental work. SS, SA, GM, KRY and PPS contributed to chemical synthesis and structure activity relationship analyses. GA and RS contributed to the molecular modeling and docking studies. SR contributed to mutant study. NK performed the

\section{Declaration of Interests}

495 The authors declare No competing interest. 


\section{Figure Legends}

Fig. 1 Nef directly interacts with the cytoplasmic tail peptides of CD80/86.

a) Illustration shows the CD80 and CD86 receptors with their extracellular,

(LWKWKKKKRPRNSYKCGTNT, 267-286 a.a) is highlighted within the cytoplasmic

tail domain (b) Graph shows direct binding of Nef to CD80 as measured by

Microscale scale thermophoresis (MST). CD80 peptide was titrated from $950 \mu \mathrm{M}$ in reaction volume. $A$ curve with upper saturation with $\mathrm{kD}=27 \mu \mathrm{M}$ was obtained; $\mathrm{x}$ axis= peptide concentration $(\mathrm{nM})$ and y axis= percentage normalized fluorescence ( $\Delta$ Fnorm); Plots represent the mean \pm SD (error bars) from three independent experiments (c) Similarly, CD86 peptide was titrated from $10 \mu \mathrm{M}$ with a 2-fold serial dilution upto 16 points against a fixed Nef concentration (35 nM). A sigmoidal curve CD86 cytosolic peptides binds to Nef. The OD measurement was done at 450nm.

516 CD74, a negative peptide control shows minimal OD value (e) Graph shows

517 Normalized Percentage Index (NPI) on a normal distribution curve for statistical

518 significance of active compounds across qualified plates showing CD80 actives. X

519 axis= the number of compounds screened in ELISA assay; $y$ axis= normalized

520 percentage Inhibition of compounds; of Z-factor $>0.5$ analysis was used to qualify the plates. Compounds with NPI $>30 \%$ for CD80 was considered as hits (f) Similarly, 
522 NPI normal distribution curve for CD86 with Cutoff percentage for CD86 NPI>20\%

523 was considered as hits (g) Scheme shows the hits belonging to 9 scaffolds that

524 were identified in the primary screen (h) Dose response curve of a hit compound

525 from "AP" scaffold; $x$ axis= log concentration of compounds; $y$ axis= Normalized

526 percentage Inhibition, (inset) Structure of AP5 compound and its molecular

527 properties

528 Fig. 2: Cell based assay screening of active compounds from ELISA.

529 (a) Nef mediated down-regulation of surface CD80 or CD86 in 3 different cell lines as

530 indicated. FACS data showing the normalized surface levels of CD80; $I_{x} / I$ (y axis)

531 where $I_{x}$ is the average fluorescence intensity in the indicated condition (from a

532 triplicate) and I is the Median of normalized negative control (No Nef control) (b)

533 FACS data shows restoration of CD80 receptors in RAJl cell line after pre-treatment

534 with 3 representative compounds AP5, PA4 and BC5 at 10 and $100 \mu \mathrm{M}$ for $24 \mathrm{~h}$ and

535 analysis after $2 \mathrm{~h}$ post Nef protein delivery (c) RAJl cell line was infected with viral

536 particles (Nef-YFP and YFP alone control cells) in viral infection assay and surface

537 CD80 receptors with compounds were measured by flow cytometry (d) Effects of

538 inhibitors on Nef-MHC-I interactions. RAJl cells were treated with compounds at 100

$539 \mu \mathrm{M}$ and then stained with anti-MHC-I antibody. MHC-I was detected by flow

540 cytometry and shown as $\mathrm{I}_{\mathrm{x}} / \mathrm{I}$ plots. Compound AP5 shows no restoration of MHC-I

541 indicating its specificity for the Nef-CD80 interface.

542 Fig. 3: Restoration of functional T-cell activation in a viral infection assay

543 (a) Schematic of a functional assay for screening of compounds that disrupt Nef-

544 CD80/86 interactions in a virally-infected cell. Functional T-Cell activation is based

545 on APC-T cell co-cultures. The APC has CD80/86 and concurrent presence of anti-

546 CD3 antibody promotes T-cell activation (as measured via IL-2) in co-cultured T-cells 
547 (b) Graph shows cytokine release in functional T-cell activation assay where antigen

548 presenting cell RAJI (B-cells) and Jurkat-cells (T cells) were co-cultured as indicated

549 (with/without anti-CD3 antibody); T-cells alone, B-cells alone controls do not show

550 measurable IL-2 release. (c) Graph shows quantification of cytokine (IL-2) released

551 after T-cells and B-cells co-culture for $3 \mathrm{~h}$; here B-cells were pretreated with the

552 indicated concentrations $(1,10,100 \mu \mathrm{M})$ of compounds for $24 \mathrm{~h}$ followed by viral

553 infection for 96 h (d) Graph shows quantification of cytokine (IL-2) released after T-

554 cells and B-cells co-culture for $3 \mathrm{~h}$; here B-cells were first infected with virus for 96h

555 and then treated with compounds at 1, 10, $100 \mu \mathrm{M}$ for $24 \mathrm{~h}$. IL-2 release $(\mathrm{pg} / \mathrm{ml})$ was

556 determined by ELISA by plotting against an IL-2 standard curve. Note: viral infection

557 reduces IL-2 release, and all 3 compounds showed a dose dependent restoration of

$558 \quad \mathrm{IL}-2$ release. AP5 showed IL-2 release at $1 \mu \mathrm{M}$.

559

Fig. 4: Modelling of Nef with multi-template computational approach

560 (a) Cartoon representation of the predicted structure of Nef shows flexible N-terminal

561 region and well-conserved core domain, colored in accordance with their position ( $\mathrm{N}$ -

562 terminal in blue to $\mathrm{C}$-terminal in red) with respective $\alpha$-helices and $\beta$-sheets as

563 indicated. (b) The ab initio shape of the solution structure of the Nef (blue color) from

564 the SAXS data (grey surface) fits well with the computational model (cartoon

565 representation) (c) Surface representation of HIV-1 Nef is depicted with the best

566 CD80 binding pose. CD80 peptide in a docked pose (cyan color) in Nef obtained

567 using SiteMap program. The inset shows the important residues of Nef involved in

568 interaction with CD80 at Sites 1 and 2.

569 Fig. 5: Evaluation of interaction between CD80 and Nef mutants in biochemical

570 and cell-based assays

571 (a) Graph shows colorimetric signal of immobilized CD80 cytosolic peptide upon 
binding to $\mathrm{Nef}^{\mathrm{WT}}$ or Nef mutants as measured by ELISA at OD450nm. Two mutants

Nef ${ }^{\mathrm{K} 99 \mathrm{~A}}$ and Nef ${ }^{\mathrm{R} 111 \mathrm{~A}}$ showed reduced affinity to CD80 peptide (b) Graph shows

574 FACS data of surface levels of CD80 receptors in RAJl cell line after delivery of

575 Nef ${ }^{\mathrm{WT}}$ or Nef mutant protein delivery. No significant down regulation seen with

576 mutants Nef ${ }^{\mathrm{K} 99 \mathrm{~A}}$ and $\mathrm{Nef}^{\mathrm{R} 111 \mathrm{~A}}$ (c) Graph shows the levels cytokine (IL-2) released in

577 supernatants of cells in the co-culture functional T-cell activation assay after delivery

578 of the Nef mutants as compared to the wild type Nef protein. (d) Graph shows FACS data of $\mathrm{MHC}-1$ levels after delivery with $\mathrm{Nef}^{\mathrm{WT}}$ and mutants. Nef ${ }^{\mathrm{WT}}$ or mutants were delivered into RAJI cells using Chariot ${ }^{\mathrm{TM}}$ delivery reagent. MHC-I was detected by

581 flow cytometry and shown as Ix/I plots.

Fig. 6: Structural and functional evaluation of the interaction between Nef and

(a) Structural and functional evaluation of the interaction between Nef and AP5The binding site of AP5 molecule overlaps with the CD80 binding site (Site-1). The inset shows the important residues for the interaction between AP5 and Nef. The nonpolar residues such as $W_{61}, L_{91}, l_{109}$ and $L_{115}$ contribute to hydrophobic interactions with $\mathrm{CF}_{3}$. AP5 ligand docking studies shows that the binding interactions occurs between the $\alpha 4$ and $\alpha 5$ helices along with few residues such as $W_{61}, E_{65}$ and $R_{111}$ which are crucial for AP5-Nef interaction (b) Graph shows colorimetric signal of

592 immobilized CD80 cytosolic peptide upon binding to Nef ${ }^{\mathrm{WT}}$ or Nef mutants in the presence /absence of $10 \mu \mathrm{M}$ AP5 as measured by ELISA at OD450 nm. (g) Graph

594 shows surface levels of CD80 receptors in RAJl cell line after the delivery of Nef'WT or

595 Nef mutant protein delivery as measured by FACS in the presence /absence of 10 $\mu \mathrm{M}$ AP5. Nef ${ }^{\mathrm{W} 61 \mathrm{~A}}, \mathrm{Ne}^{\mathrm{K} 99 \mathrm{~A}}$ and $\mathrm{Ne}^{\mathrm{R} 111}$ did not show any further change in CD80 
597 levels with AP5 addition. (c) Graph shows cytokine (IL-2) release in supernatants

598 after the co-culture T-cell activation assay. RAJl cells were pre-treated with $10 \mu \mathrm{M}$

599 AP5 for $1 \mathrm{~h}$ and then the cells were delivered with Nef mutants or wild type Nef

600 protein for $2 \mathrm{~h}$ before co-culture with Jurkat T-cells for $3 \mathrm{~h}$. The IL-2 levels remain

601 unchanged with and without addition of AP5 compound in all three mutants NefW61A,

$602 \mathrm{Nef}^{\mathrm{K} 99 \mathrm{~A}}$ and Nef ${ }^{\mathrm{R} 111}$. Reduction in IL-2 seen with mutant Nef ${ }^{\mathrm{E} 160 \mathrm{~A}}$ comparable to

603 NefWT.

604 Fig. 7: SAR and Hit refinement with AP5 as a template (a) Scheme shows

605 Medicinal chemistry approach for hit refinement of AP5 showing two series of

606 compounds (b) Summary of SAR strategy to design compounds similar to AP5

607 structure with modifications made on rings $A, B$ and $C$ as indicated. The

608 synthesized molecules were evaluated for their effect on Nef-CD80/CD86 inhibition

609 (c) Heat map table showing SAR with synthesized compounds. The various

610 substitutions in rings $A, B$ and $C$ are indicated as well as their activity in the ELISA

611 and cell-based assays

612 


\section{STAR methods}

Materials and Reagents:

616 96-well maxisorp ELISA plates (NUNC, cat\#449824,), small molecule inhibitors

617 (synthesized by IIIM, Jammu), DMSO (Sigma, cat\#D2650), Anti-Nef Antibody (from

618 ICGEB), DAR-HRP (Jackson Immunoresearch, cat\#711-035-152), Tecan ELISA

619 reader. The following commercially synthesized peptides (Peptron Inc., South Korea)

620 were used: CD80 cytosolic tail peptide: PRCRERRRNERLRRESVRPV (20-mer), CD86 cytosolic tail peptide: LWKWKKKKRPRNSYKCGTNT (20-mer), CD74, a nonspecific peptide: MHRRRSRSCREDQKPVMDDQRDLISNNEQL (30-mer). Nef construct was cloned at ICGEB, rF2-Nef protein (HIV-1 Subtype C) cloned into pET28 vector (Novagen, cat\#69865) at Ncol and Xhol sites with 6XHis tag at Cterminal end. Nef and all Nef variant proteins were expressed in E. coli Rosetta strain containing pRARE that codes for t-RNAs corresponding to rare Arginine codons in the bacterium. The growth and/or expression medium used were Luria chloramphenicol $(25 \mu \mathrm{g} / \mathrm{ul})$ for pRARE selection, IPTG induction, Akta FPLC -Affinity and Size exclusion chromatography for protein purification.

Cell lines- RAJI B lymphocytic, Burkitt's lymphoma cell line (NIH AIDS reagent cat\#ARP-9944), Jurkat T cells (ATCC, cat\#TIB-152), for cell-based assays,

634 of viral particles.

635 Cell culture reagents- RPMI 1640, FBS, glutamine, Penstrep (from Gibco),

636 Chariot $^{\mathrm{TM}}$ delivery reagent (active motif, cat\#30100) was used for protein delivery

637 into cells. Viafect (Promega, cat\#E4982) reagent was used for DNA transfection, 638 lentiX concentrator (clontech, cat\#631231), lentiblast reagent (OZ 
biosciences,cat\#LB00500), DMEM (Gibco), WST-1 reagent (Roche), anti-CD80 and

644 (Qiagen), biotinylated anti-human MHC-I (HLA-A, B, C) antibody (biolegend, cat\#311402).

\section{Chemical Compound Synthesis}

647 The 25 active hits belong to three scaffolds namely amino pyrimidine (AP), biaryl

648 (heteroaryl) carbamate (BC) and phenoxy acetamide (PA) has been selected and details regarding the strategies applied for the synthesis of hits and hit optimized compounds has been provided in Scheme 1-8 (Supplementary data). synthesized and their synthetic strategy has been given in Scheme 1 and 2. The synthesis started with commercially available 2-chloro-5-bromopyrimidine $\mathbf{1}$ as the starting material which was treated with ethanethiol 2 afforded intermediate $\mathbf{3}$ (Scheme 1). The intermediate 3 was then subjected to Suzuki coupling with aryl boronic acid $\mathbf{4}$ provided 5 -aryl pyrimidine $\mathbf{5}$. The compound $\mathbf{5}$ was then oxidized with $m$-CPBA to corresponding sulfoxide 6 followed by nucleophilic substitution afforded key intermediate 7, which on reaction with acetylacetone provided AP1. In the next attempt, the intermediate 7 on reaction with substituted aldehydes provided AP2 and AP3. In another attempt, the 2-chloro-5-bromopyrimidine 1 was converted into AP4

661 in two steps i) nucleophilic substitution with dimethyl amine; ii) Suzuki coupling with

662 phenyl boronic acid. The synthesis of AP5 required the quite different strategy and is shown in Scheme 2. The synthesis started with commercially available resorcinol 11 
which was undergoes acetylation followed by reduction gave intermediate 13 . The intermediate 13 was treated with 4-methoxyphenyl acetonitrile 14 to get acylated intermediate 15 which on treatment with trifluoroacetic anhydride underwent cyclization to generate the chromone based key intermediate 16 followed by the methylation of hydroxyl group to get $\mathbf{1 7}$, which on reaction with guanidine hydrochloride to afford the hit AP5.

670 For the initial hit refinement, we started synthesis with commercially available methodology was developed in the presence of light and $\mathrm{K}_{2} \mathrm{~S}_{2} \mathrm{O}_{8}$ gave intermediate 29. Intermediate $\mathbf{2 9}$ was brominated in the presence of $N$-bromosuccinamide (NBS) provided the intermediate $\mathbf{3 0}$. The final targeted compounds AP(S1-S4) were synthesized in good to moderate yields from the reaction of compound $\mathbf{3 0}$ with substituted phenyl and heterocyclic boronic acids $\mathbf{4}$ under Suzuki conditions to provide final compounds AP (S1-S4, scheme-3). For series-2, sequence of synthesis began with 2-amino-4-chloropyrimidine 31 which underwent Suzuki coupling reaction with un/substituted aryl and heteroaryl boronic acids 4 to get intermediate 32 which on reaction with NBS provided intermediate $\mathbf{3 3}$. The brominated intermediate $\mathbf{3 3}$ was subjected to Suzuki couplings with a range of aryl and heteroaryl boronic acids $\mathbf{4}$ to provide final compounds AP (S5-S10, Scheme-4).

683 In the case of biaryl (heteroaryl) carbamate (BC), seven hits were synthesized 684 (Schemes 5-6). The synthesis started with commercially available phenylchloroformate 18 which on treatment with benzo[d]oxazole-2(3H)-thione 19 in the presence of base to give the hit BC1 (Scheme 5). All other hit molecules from 
chloroformates 18 reacted with substituted anilines 20 to afford the targeted hits

689 (Scheme 6).

690 In case of phenoxyacetamide (PA), syntheses of eight hits were accomplished as

691 outlined in Scheme 7 and 8. Hit molecules like PA2, 3, 5, 6 and 7 (Scheme 7) were

692 synthesized in two steps. i) by treating substituted anilines $\mathbf{2 0}$ with different

693 chloroacetyl chloride in the presence of base at room temperature followed by; ii) coupling with substituted phenols 23. For the synthesis of hits PA1, $\mathbf{8}$ and 10, the synthesized involves four steps. The substituted phenols 23 coupled with substituted 2-chloroethylacetate $\mathbf{2 4}$ to form intermediate $\mathbf{2 5}$, which on hydrolysis gave intermediate 26 . The intermediate 26 was converted into corresponding aryl chloride $\mathbf{2 7}$ and then coupled with substituted anilines $\mathbf{2 0}$ to get the desired hits PA1, 8 and 10 (Scheme 8).

The identified hits such as BC2, 6 and 8, and PA4 and PA9 were not synthesized and were procured in somewhat large quantities from the original commercial vendors because of the unavailability of the starting materials. These hits were characterized by using NMR and Mass spectroscopy and then were taken up for validation study.

705

Compound stock and storage- All compounds were dissolved $100 \%$ DMSO to make a $10 \mathrm{mM}$ stock. Multiple aliquots were prepared from mother stock to avoid

707 multiple freeze-thaw cycles and were stored at $-80^{\circ} \mathrm{C}$

\section{Expression and Purification of recombinant Nef}

HIV-1 Nef gene sequence was cloned into pET28b expression vector with antibiotic resistance to chloramphenicol $(25 \mu \mathrm{g} / \mathrm{ml})$ and kanamycin $(50 \mu \mathrm{g} / \mathrm{ml})$ and recombinant

711 Nef-His tag protein was expressed in E. coli Rosetta strain [OD600 0.5-0.6]. IPTG 712 induction $(0.2 \mathrm{mM})$ was done for $4 \mathrm{~h}$ at $28^{\circ} \mathrm{C}$. The cells were spun down at $10000 \mathrm{rpm}$ 
713 for 15 minand pellet stored at $-80^{\circ} \mathrm{C}$. The protein was purified in $20 \mathrm{mM}$ Tris $\mathrm{HCl}$,

714 150mM, NaCl, 3 mM DTT, 5\% glycerol, 0.2\% Tween-20 in a Ni-NTA column and gel

715 filtration chromatography on Sepharose-75pg (GE) in Akta FPLC purifier. Nef protein

716 with single mutants W61A, K99A, R111 and E160A were designed by site-directed

717 mutagenesis. The buffer conditions were same as full length WT-Nef.

\begin{tabular}{|c|c|}
\hline Mutant & Primers used \\
\hline W57A & F: GAT TGT GCT GCG CCG GAA GCG C \\
\hline & R: GCG CTT CCG GCG CAG CAC AAT C \\
\hline K94A & F: AAA GAA GCG GGG GGA CTG GAA GGG \\
\hline & R: CCC TTC CAG TCC CCC CGC TTC TT \\
\hline R106A & F: CTAAGAAAGCGCAAGAGATCCTTGATTG \\
\hline & R: CAAATCAAGGATCTCTTGCGCTTCTTAG \\
\hline E155A & F: GCA GTA GAA GCG GCC AAC GAA GGA G \\
\hline & R: CTC CTT CGT TGG CCG CTT CTA CTG C \\
\hline
\end{tabular}

\section{Microscale Thermophoresis (MST)}

720 Full length Nef was labeled with lysine NT-647-NHS fluorescent dye using the

721 Monolith NT.115 Protein Labeling Kit (NanoTemper Technologies). A Capillary

722 Scanning was performed to check the optimal fluorescence intensity of the labelled

723 protein for titration with the ligand. For the direct binding assay, a final concentration of labelled 35nM Nef protein was titrated against a 16-point 2-fold serial dilution series starting from $950 \mu \mathrm{M}$ for CD80 peptide and for CD86 a final concentration of labelled 25nM Nef protein was titrated against a 16-point 2-fold serial dilution series starting from $10 \mu \mathrm{M}$. The compound was titrated against the protein-peptide at16-

728 point dilution. All samples were prepared by centrifuging at $10000 \mathrm{rpm}$ for $5 \mathrm{~min}$ at

$7294^{\circ} \mathrm{C}$ and $10 \mu \mathrm{l}$ of the supernatant was loaded into premium glass capillaries

730 (NanoTemper Technologies). MST runs were performed at MST power of $60 \%$ and excitation power of $50 \%$, using a Monolith NT.115 NanoTemper Technologies. The

732 data was analyzed using NanoTemper analysis softwareMO. Affinity Analysis v2.2.4.

$733 \mathrm{Kd}$ values were determined using T-jump and thermophoresis settings. The change 
in thermophoresis between each sample dilution was represented as normalized fluorescence $\left(\Delta F_{\text {norm }}\right)$, which is defined as $F_{\text {hot }} / F_{\text {cold }}$, where $F_{\text {cold }}$ is the control and Fhot is the experimental condition. The binding kinetics to non-fluorescent ligand causes a change in thermophoresis which is determined by area in the curve under steady-state conditions to yield a binding curve.

\section{Primary screening by Indirect Enzyme-linked immunosorbent assay (ELISA)}

An indirect ELISA was performed to measure the interaction of Nef-CD80/CD86 and their disruption with addition of compounds.CD80, CD86 and a non-specific control peptide derived from the cytoplasmic tail of CD74 peptides were separately immobilized at $10 \mu \mathrm{M}$ concentration onto 96-well micro-titer plate and incubated overnight at $4{ }^{\circ} \mathrm{C} .5 \%$ blotto was used as blocking buffer to reduce the background interference. Blotto was completely removed with PBS-Tween (0.1\%) washes. 10 $\mu \mathrm{M}$ of Nef protein and $10 \mu \mathrm{M}$ of compounds were pre-incubated at $\mathrm{RT}$ for 1 hour and added onto peptide coated wells for 1 hour incubation. Compounds were diluted in $2.5 \%$ blotto and were screened at a concentration of $10 \mu \mathrm{M}$. After each addition step PBS-T washes were done. DMSO in $2.5 \%$ blotto as vehicle control and plate background (no coating) were used as negative control. In subsequent steps the plates were incubated with primary anti-Nef antibody and secondary antibody with HRP. The TMB substrate was added which reacts with HRP to produce a coloured product within 15 minutes. The reaction was stopped with $1 \mathrm{~N}$ sulphuric acid and the absorbance at 450nm was recorded with Tecan infinite 200 PRO plate reader. MATLAB 7.5 program was used to pick the active compounds. The program assessed the robustness of the screened plate using Z-factor (for qualifying plate) and $Z$ score for active compounds, and Normalized percent inhibition to determine the compound activity. 


\section{Robustness of screen}

760 The plate controls showing a $Z$ factor $>0.5$ was considered to qualify for further analysis. The activity of compounds was determined with the Normalized Percent Inhibition (NPI) and Z scores were used to assess the efficacy of the compounds in the screen: Z-score: $(\mid x-\mu D) / \sigma D$

Where, IX: Intensity of triplicate x (first, second or third) represented as either A/B/C

$\mu \mathrm{D}$ : Mean of negative control

$\sigma D:$ Standard deviation of triplicates of negative control

The compounds identified as 'hits' in primary screen were defined as those two of three repeats of a particular compound should have Z score between - 2 and -

8.

Determination of $I_{50}$ for active compounds- The active compounds from primary screen were tested for dose response with 10-fold dilution for an 11-point curve from $10 \mu \mathrm{M}$ to nanomolar concentration. Compounds showing an $\mathrm{IC}_{50}$ in nanomolar concentrations were further tested in cell-based assay.

\section{WST assay for quantification of cell viability and toxicity}

77750000 cells per well were seeded in a 96 well plate. $1 \%$ Triton X (control) and compounds were treated at $100 \mu \mathrm{M}$ in triplicates in $100 \mu \mathrm{l}$ media and incubated for the 24 hours' timepoint. $10 \mu \mathrm{l}$ of WST-1 reagent per well was added and incubated for $3 \mathrm{hrs}$.Absorbance read at $450 \mathrm{~nm}$ with reference $620 \mathrm{~nm}$. The media alone control was used to normalize all the wells. The cells alone control was the high control and triton treated cells were the low control or negative control. 
(Average $(x)$ - Average (high control) / Average (low control) - Average (high control) )*100

Where, Average $(\mathrm{X})=$ average $\mathrm{OD}$ of individual test; Average $($ high control) $=$ average OD

\section{Measurement of surface levels of CD80/CD86 receptor by flow cytometry}

Nef protein was delivered into RAJI B cells using Chariot ${ }^{\mathrm{TM}}$ protein delivery reagent according to manufacturer's protocol (Active Motif). In brief, $1.5 \times 10^{5}$ cells were layered with protein-delivery reagent complex, incubated and complete media

791 (RPMI+10\% FBS) was added. The cells were then harvested and stained with CD80

or CD86 biotinylated antibody or isotype control followed by Streptavidin-APC at $4^{\circ} \mathrm{C}$ and flow profile acquisition was done on Gallios Flow Cytometer (Beckman Coulter).

Data was analyzed by FlowJo LLC software. Surface levels of CD80 and CD86 was calculated by normalizing raw fluorescent measurements relative to controls (Normalized Inhibition of Down-regulation). To test compounds, two concentrations 10 and $100 \mu \mathrm{M}$ were pre-treated on cells for 1 hour and then $50 \mu \mathrm{g}$ of Nef was delivered with method described and quantified by flow cytometry.

The potential actives from the plates were selected based on the following metrics.

a) Z score:

Z-score: $\frac{I_{X}-\mu_{D}}{\sigma_{D}}$

801 Where $I_{x}$ is the measurement of each triplicate, $\mu \mathrm{D}$ : mean of population and $\sigma \mathrm{D}$ :

802 Standard deviation of population (excluding the positive and negative control). Z

803 score was used to select hits reversal of CD80/86 downregulation by Nef.

804 Compound repeats were qualified by $Z$ score selection, with a $Z$ score between 1.5

805 to -1.2 . Each graph was calculated with student t-test using Graphpad prism $7.0\left({ }^{*} \mathrm{p}\right.$ 
$\leq 0.05 ;{ }^{* *} p \leq 0.01 ;{ }^{* *} p \leq 0.001$ ) was used to determine the significant difference between the means of control group and treatment groups.

Calculation of surface CD80 and CD86 down regulation- was calculated by

normalizing raw fluorescent measurements relative to controls (Normalized Inhibition

of Down-regulation)

$$
\mathrm{NID}=\mathrm{I}_{\mathrm{x}} / \mathrm{I}
$$

812 Where $\mathrm{I}_{\mathrm{x}}$, is the raw measurement each triplicate and $\mathrm{I}$ is the mean of the measurements on the positive control.

\section{Virus particle generation}

815 HEK293T transfection: The 4 retroviral components present in the following plasmids, pVSV-G (envelope), p-gag-pol (packaging), pMSCV-Nef-YFP (HIV-1 F2

817 isolate-based retroviral clones harboring nef sequence), pMSCV-YFP (control) were isolation (miniprep Qiagen). The plasmid concentration was checked by

820 NanoDrop ${ }^{\mathrm{TM}}$.

821 Virus particle generation: To generate recombinant retroviral particles, HEK293T were seeded into $100 \mathrm{~mm}$ dish with $5 \times 10^{6}$ cells on day 1 . At $80 \%$ confluency, Viafect TM transfection reagent with $15 \mu \mathrm{g}$ of DNA vectors consisting of $\mathrm{p}-\mathrm{gag}$ pol, $\mathrm{pVSV}-\mathrm{G}$ and YFP or Nef-YFP in 3:1:4 ratios was layered over cells with DMEM (5\% FBS).

825 The cells are incubated for $72 \mathrm{~h}$. The cells were observed under fluorescence

826 microscope for YFP expression to estimate the percentage transfection. The cell supernatant was collected and concentrated with Lenti- $\mathrm{X}^{\mathrm{TM}}$ reagent (as per manufacturer's protocol). The visible pellet was reconstituted with minimal volume of

829 PBS (1X) to make a concentrated viral stock. The stock was titrated in 3-fold serial 830 dilution onto HEK293T $\left(1 \times 10^{5}\right)$ cells/ well in a 24 well plate. Polybrene $(8 \mu \mathrm{g} / \mathrm{ml})$ was 
added to cells along with DMEM (5\%FBS) media. After $72 \mathrm{~h}$, the cells were harvested and analyzed on Gallios flow cytometer. The fluorescent population was gated and analyzed against the cells alone. The infection percentage above $30 \%$ and below $0.5 \%$ is omitted. The higher titer tends to be underestimated; lower titer falls too close to the background. The average of the titer was used to calculate the viral units present in the stock using the low formula:

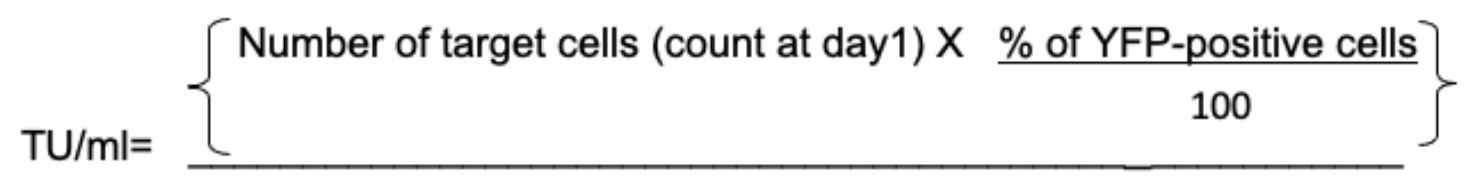

\section{Volume of supernatant in $\mathrm{ml}$}

Functional T-cell activation Assay - RAJl cells were infected with MOI-0.02 of viral stock of control YFP virus or Nef-YFP containing virus and incubated in a 24-well cell bind plate. Additionally, LentiBlast ${ }^{\mathrm{TM}}$ reagent $A$ and $B$ was added in 1:1 ratio to the cells and incubated for 48 to $96 \mathrm{~h}$. The cells showed YFP signal post infection. The cells were harvested and stained for CD80 and CD86 and analyzed by flow cytometry and the percentage down-modulation of the receptors was calculated with median values.

Virus infected RAJI- cells was co-cultured with Jurkat T cells (1:1 ratio, $2.5 \times 10^{5}$ cells) in the presence of $0.06 \mu \mathrm{g} / \mathrm{mL}$ of anti-CD3 antibody (OKT3 clone, BioLegend®) in final volume of $200 \mu \mathrm{l}$ and incubated for $3 \mathrm{~h}$ for $37^{\circ} \mathrm{C}$ in a 96 well plate and the supernatant was harvested by centrifuging twice at 4000 rpm. IL-2 Cytokine release in the supernatant was quantified by BioLegend® kit-based ELISA method and ODs were measured in Tecan infinite 200 PRO plate reader. 
Testing of compounds in viral assay for functional T-cell activation assay- Pretreatment with compounds AP5, PA4 and BC5 at 1, 10 and $100 \mu \mathrm{M}$ concentrations cells were pre-treated with $0.5 \%$ DMSO. Post-treatment of compounds for $24 \mathrm{~h}$ was done after $96 \mathrm{~h}$ of viral infection.

\section{Staining of surface MHC-I antibody}

858 RAJl cells were pre-treated with compounds AP5, PA4 and BC5 at $100 \mu \mathrm{M}$ and 50 $\mu \mathrm{g}$ Nef was delivered with Chariot $^{\mathrm{TM}}$ reagent. The cells were stained for receptors with anti-human MHC-I biotinylated (HLA-A, B, C, clone W6/32) BioLegend® antibody and secondary streptavidin APC and was run on Gallios flow cytometer and the surface levels MHC-I was quantified. The percentage down regulation recovery of $\mathrm{MHC}-\mathrm{I}$ receptors was analyzed.

To build a full-length Nef protein, multi-template modelling approach was performed, where more than one experimentally determined structure was utilized for building the model. For the N-terminal part, NMR structure of Nef anchor domain(1QA5) and for core domain, the NMR structure of HIV-1 Nef (2NEF: A) and X-ray structure HIVtemplates. Among the three templates, major structural information is acquired from 2NEF structure which covers maximum region of core domain. The tool

872 MODELLER7 (version 9v8) was used to obtain the full-length Nef model. After modeling, the lowest energy state structure was further energy minimized through

874 SYBYL (Version 7.1) (Tripos Associates Inc.) and validated using PROCHECK ${ }^{46}$ 
877 The modeled full-length Nef protein is utilized for examining the potential-ligand

878 association site using the SiteMap ${ }^{c}$ tool in Schrödinger software. SiteMap identifies

879 potential peptide/ligand binding sites considering van der Waals forces and hydrogen

880 donor/acceptor characteristics. SiteScore is the most important property generated

881 by SiteMap, proven to be effective at identifying possible binding sites in 3D

structure. The prediction of the binding site is based on set of properties such as size

of the site, degrees of enclosure by the protein and exposure to solvent, tightness

884

with which the site points interact with the receptor, hydrophobic and hydrophilic character of the site. The sitemap predictions are useful in identifying the possible binding sites of CD80/CD86 cytoplasmic tails.

887 Further, docking studies were performed to identify the interactions of Nef protein with co-stimulatory molecules CD80/CD86. This was achieved by modelling of cytoplasmic regions of CD80/CD86 using I-TASSER server and protein-peptide docking using BioLuminate module in Schrödinger software. Though numerous information about the Nef interaction sites with other cell surface receptors are available, the peptide is docked by blind docking approach where no guidance about residues, that could potentially participate in interaction with CD80 was provided to the program. The best docked pose is selected by energy minimization followed by implicit solvent based energy calculations.

The protein-peptide docking resulted in 30 best poses of Receptor-ligand complexes.

897 The predicted poses are ranked based on the maximum number of occurrences of 898 that particular pose. Since there is no prior information about the binding pattern of

899 Nef with CD80/CD86, it is necessary to score each pose based on energy

900 calculations. The association of the protein-peptide complex is estimated by an

901 automated mechanism of Multi-Ligand Bimolecular Association with Energetics 
(eMBrAcE) (MacroModel, version 9.6, Schrödinger, LLC, New York, NY, 2008). The best identified Nef-CD80 binding pose analysed further for important amino acids

904 involved in the non-bonded interactions which is contributing for binding. In order to validate the predicted binding mode, best binding small molecule AP5 was docked with the full-length Nef model. This was basically achieved by selection of a centroid point from the predicted Nef-CD80 binding site using Glide docking protocol from examined for the non-bonded interactions and best docked score.

\section{SAXS data collection and analysis}

911 SAXS-data of the apo HIV1-Nef was measured with the BIOSAXS-1000 small-angle X-ray scattering with Kratky camera system, installed on a Rigaku microfocus X-ray generator (1.5418 $\AA$ wavelength). The purified HIV1-Nef at 1,3 and $5 \mathrm{mg} / \mathrm{ml}$ concentrations were used with buffer containing $50 \mathrm{mM}$ Tris/HCl, $\mathrm{pH}$ 7.5, $200 \mathrm{mM}$ $\mathrm{NaCl}$ in a sample volume of $60 \mu \mathrm{l}$ inside a vacuum tight quartz capillary subjected $\mathrm{X}$ rays at $25^{\circ} \mathrm{C}$. The data was collected for $30 \mathrm{~min}$ and for each measurement a total of six frames at 5 min intervals were recorded. Corresponding to each protein sample, data were collected for a buffer under identical experimental conditions, providing a background scattering curve. The data was then tested for possible radiation damage by comparing the six data frames and no changes were observed. The scattering of

921 the buffer was subtracted from the scattering of the sample. All the data processing 922 steps were performed using the program package PRIMUS ${ }^{47}$. The experimental data obtained for all protein samples were analyzed for aggregation using the Guinier region. The forward scattering $\mathrm{I}(0)$ and the radius of the gyration, $\mathrm{Rg}$ were computed using the Guinier approximation assuming that at very small angles $(q<1.3 / \mathrm{Rg})$ the 926 intensity is represented as $\mathrm{I}(\mathrm{q})=\mathrm{I}(0) \exp (-(q \mathrm{Rg}) 2 / 3)$. These parameters were also 
927 computed from the extended scattering patterns using the indirect transform package

928 GNOM, which provides the distance distribution function $P(r)$ of the maximum particle

929 dimension, Dmax as well as the radius of gyration, $\mathrm{Rg}$, qualitative particle motion was

930 inferred by plotting the scattering patterns in the normalized Kratky plot

$931((\mathrm{qRg}) 2(\mathrm{I}(\mathrm{q}) /(\mathrm{l}(0))$ vs $\mathrm{qRg})$. Ab initio low-resolution models of the proteins were built by

932 the program DAMMIF ${ }^{48}$ considering low angle data $\left(\mathrm{q}<2 \mathrm{~nm}^{-1}\right)$. Ten independent $a b$

933 initio reconstructions were performed for each protein and then averaged using

934 DAMAVER ${ }^{49}$. Superimposition between ab initio reconstruction and atomic model was

935 performed using the software SUPCOMB ${ }^{50}$.

936 Statistical Analysis: $Z$ factor was used for qualifying the plate and t-test was used

937 for determining the significant data. The plots were prepared using Graph Pad Prism 938 Ver6.0.

939

940 


\section{Supplementary Information}

\section{Supplementary figure legends}

943 Fig. S1: a) Sequence of Subtype B and C with overlapping residues and important

944 residues highlighted (in red) (b). Sequence of full length CD80 and CD86, where the

945 20-mer cytoplasmic peptide used for screening assays are highlighted (in cyan) (c)

946 Schematic of ELISA procedure in microwell plate where the CD80 and CD86

947 peptides were immobilized. The Nef protein was incubated with the peptide and their

948 interaction was detected by anti-Nef antibody and secondary antibody with HRP. The

949 colorimetric signal was quantified with TMB reducing the HRP substrate

950 Fig. S2: Detailed chemical synthesis of the hits belongs to the AP, PA and BC

951 scaffolds. Note: Details of Characterization of Synthesized compounds and intermediates will be included in the full submission.

953

Fig. S3: Regression graph showing correlation with replicate data $\mathrm{R}^{2}$ values for hit

954 compounds in biochemical screen, where $\mathrm{plC}_{50}$ is the negative log of $\mathrm{IC}_{50}$

955 expressed in molar units.

956 Fig. S4: Graph shows cytotoxicity profile with treatment of compounds. RAJI cell line

957 was treated at the highest concentration of $100 \mu \mathrm{M}$ for $24 \mathrm{~h}$ and the supernatant

958 collected was estimated for WST assay.

959 Fig. S5: FACS histogram shows surface staining of CD80 and CD86 with APC-

960 tagged specific CD80 or CD86 antibody. Colour representation: Unstained Cells (in 961 red), Isotype controls -IgG1k for CD80; IgG2b for CD86 (in blue dotted line), cells with Vehicle control Chariot reagent showing CD80 or CD86 surface expression (in orange), Protein delivered (in green). The reduction in CD80/CD86 levels are seen

964 with Nef protein after its delivery into RAJI cells in $2 \mathrm{~h}$ incubation period. The surface 
levels of CD80 did not change with the delivery of $100 \mu \mathrm{g}$ of Ovalbumin and $\beta$ lactoglobulin proteins with Chariot $^{\mathrm{TM}}$ reagent.

Fig. S6: Schematic of the reterovirus used for infection assays. Distribution of regulatory elements in the HIV-1. (A) Schematic depiction of the HIV-1 genome containing accessory $v p r, v p u$, and nef genes.

(B) Schematic depiction of reteroviral vector with Nef transgene. where Env protein used in this reterovirus is $\mathrm{VsVg}$ from pMLV, and Vpr, Vif and Vpu are not present in this vector.

Fig. S7: a) SAXS-patterns of full length HIV1-Nef at three different concentrations 1, 3 and $5 \mathrm{mg} / \mathrm{ml}$ are shown. The Guinier plots at low angles appeared linear and showed no aggregation (shown in inset) (b) The Rg, Dmax and molecular mass of the full length HIV1-Nef suggest the progressive increase in the Rg, Dmax and molecular mass values with increasing concentration of Nef. The deviation from a typical bell-shaped profile depicts an inherent structural flexibility of Nef Protein (c)

978 The averaged ab initio model (surface representation) overlay with the crystal 979 structure of folded C-terminal core (cartoon representation).

980 Fig. S8: a) SDS-PAGE run with purified Nef proteins. Nef wildtype and mutants show bands at $\sim 29 \mathrm{kDa}$ (b) FPLC purification profile of Nef proteins purified (c) Western intensity of Nef ${ }^{\mathrm{W} 61 \mathrm{~A}}$ protein reduced after $5 \mathrm{~h}$ when compared to $2 \mathrm{~h}$ incubation,

984 indicating degradation of protein.

\section{Supplementary tables:}

Suppl. Table 1: a) Table shows 20 compounds selected from screen showing $\mathrm{IC}_{50} \mathrm{~S}$ 
989 Suppl. Table 2: Table showing the SAXS derived parameters such as Guinier Rg,

990 Realspace $\mathrm{Rg}$ and Dmax for the full-length Nef in solution

991 Suppl. Table. 3: Table showing the residue information about the CD80 peptide

992 docked predicted sites on Nef from SiteMap program. The important residues

993 involved in protein-protein interactions are highlighted.

994 


\section{References}

1. Tsibris, A. M. N. \& Hirsch, M. S. MINIREVIEW Antiretroviral Therapy in the Clinic $\square .84,5458-5464$ (2010).

2. Gulick, R. M. \& Flexner, C. Long-Acting HIV Drugs for Treatment and Prevention. (2019).

3. Kumar, A. \& Herbein, G. The macrophage: a therapeutic target in HIV-1 infection. Mol. Cell. Ther. 2, 10 (2014).

4. Baxter, J. et al. Global HIV-1 transmitted drug resistance in the INSIGHT Strategic Timing of AntiRetroviral Treatment (START) trial. HIV Med. 16, 7787 (2015).

5. Niessl, J. et al. Combination anti-HIV-1 antibody therapy is associated with increased virus-specific T cell immunity. Nat. Med. 26, 222-227 (2020).

6. Pollara, J., Easterhoff, D. \& Fouda, G. G. Lessons learned from human HIV vaccine trials. Curr. Opin. HIV AIDS 12, 216-221 (2017).

7. Restouin, A. et al. Protein - protein interaction inhibition ( 2P2I ) combining high throughput and virtual screening: Application to the HIV-1 Nef protein. 104, (2007).

8. Flexner, C. Modern Human Immunodeficiency Virus Therapy: Progress and Prospects. 105, 61-70 (2019).

9. Jäger, S. et al. Global landscape of HIV - human protein complexes. 481, 365-370 (2013).

10. Arhel, N. J. \& Kirchhoff, F. Implications of Nef: Host Cell Interactions in Viral Persistence and Progression to AIDS. in HIV Interactions with Host Cell Proteins (eds. Spearman, P. \& Freed, E. O.) 147-175 (Springer Berlin Heidelberg, 2009). doi:10.1007/978-3-642-02175-6_8 
11. Peter, F. HIV nef: The mother of all evil? Immunity 9, 433-437 (1998).

12. Basmaciogullari, S. \& Pizzato, M. The activity of Nef on HIV-1 infectivity. Front. Microbiol. 5, 1-12 (2014).

13. Rahim, M. M. A., Hanna, Z., Hu, C., Jolicoeur, P. \& Chrobak, P. Adult AIDSLike Disease in a Novel Inducible Human Immunodeficiency Virus Type 1 Nef Transgenic Mouse Model: CD4+ T-Cell Activation Is Nef Dependent and Can Occur in the Absence of Lymphophenia. J. Virol. 83, 11830-11846 (2009).

14. Greenway, A. L., Mills, J., Rhodes, D., Deacon, N. J. \& McPhee, D. A. Serological detection of attenuated HIV-1 variants with nef gene deletions. Aids 12, 555-561 (1998).

15. Dyer, W. B. et al. Strong human immunodeficiency virus (HIV)-specific cytotoxic T-lymphocyte activity in Sydney Blood Bank Cohort patients infected with nef-defective HIV type 1. J Virol 73, 436-443 (1999).

16. Birch, M. R. et al. An examination of signs of disease progression in survivors of the Sydney Blood Bank Cohort (SBBC). J. Clin. Virol. 22, 263-270 (2001).

17. Brambilla, A. et al. Defective nef alleles in a cohort of hemophiliacs with progressing and nonprogressing HIV-1 infection. Virology 259, 349-368 (1999).

18. Trible, R. P. et al. Allosteric Loss-of-function Mutations in HIV-1 Nef from a Long-term Non-progressor. J. Mol. Biol. 374, 121-129 (2007).

19. Das, S. R. \& Jameel, S. Biology of the HIV Nef protein. Indian J. Med. Res. 121, 315-332 (2005).

20. Swingler, S., Brichacek, B., Jacque, J. \& Ulich, C. HIV-1 Nef intersects the macrophage CD40L signalling pathway to promote resting-cell infection. 424, 213-219 (2003). 
21. Pereira, E. A. \& daSilva, L. L. P. HIV-1 Nef: Taking Control of Protein

Trafficking. Traffic 17, 976-996 (2016).

22. Roeth, J. F. \& Collins, K. L. Human Immunodeficiency Virus Type 1 Nef :

Adapting to Intracellular Trafficking Pathways. 70, 548-563 (2006).

23. Landi, A., Iannucci, V., Van Nuffel, A., Meuwissen, P. \& Verhasselt, B. One

Protein to Rule them All: Modulation of Cell Surface Receptors and Molecules

by HIV Nef. Curr. HIV Res. 9, 496-504 (2011).

24. Chaudhry, A. et al. The Nef Protein of HIV-1 Induces Loss of Cell Surface Costimulatory Molecules CD80 and CD86 in APCs. J. Immunol. 175, 45664574 (2005).

25. Chaudhry, A. et al. A Two-Pronged Mechanism for HIV-1 Nef-Mediated Endocytosis of Immune Costimulatory Molecules CD80 and CD86. Cell Host Microbe 1, 37-49 (2007).

26. Chaudhry, A. et al. HIV-1 Nef promotes endocytosis of cell surface MHC class II molecules via a constitutive pathway. J. Immunol. 183, 7611-7611 (2009).

27. Seidel, S. A. I. et al. Microscale thermophoresis quantifies biomolecular interactions under previously challenging conditions. Methods 59, 301-315 (2013).

28. Venzke, S., Michel, N., Allespach, I., Fackler, O. T. \& Keppler, O. T. Expression of Nef Downregulates CXCR4, the Major Coreceptor of Human Immunodeficiency Virus, from the Surfaces of Target Cells and Thereby Enhances Resistance to Superinfection. J. Virol. 80, 11141-11152 (2006).

29. Snanoudj, R. et al. The blockade of T-cell co-stimulation as a therapeutic stratagem for immunosuppression: Focus on belatacept. Biol. Targets Ther. 1, 203-213 (2007). 
30. Geyer, M., Munte, C. E., Schorr, J., Kellner, R. \& Kalbitzer, H. R. Structure of the Anchor-Domain of Myristoylated and Non-myristoylated HIV-1 Nef Protein. 4, (1999).

31. Barnham, K. J., Monks, S. A., Hinds, M. G., Azad, A. A. \& Norton, R. S. Solution Structure of a Polypeptide from the N Terminus of the HIV Protein Nef. 2960, 5970-5980 (1997).

32. Horenkamp, F. A. et al. Conformation of the Dileucine-Based Sorting Motif in HIV-1 Nef Revealed by Intermolecular Domain Assembly. 867-877 (2011). doi:10.1111/j.1600-0854.2011.01205.x

33. Geyer, M. \& Peterlin, B. M. Domain assembly, surface accessibility and sequence conservation in full length HIV-1 Nef. FEBS Lett. 496, 91-95 (2001).

34. Chi-Hon, L., Saksela, K., Mirza, U. A., Chait, B. T. \& Kuriyan, J. Crystal structure of the conserved core of HIV-1 Nef complexed with a Src family SH3 domain. Cell 85, 931-942 (1996).

35. Dikeakos, J. D. et al. An interdomain binding site on HIV-1 Nef interacts with PACS-1 and PACS-2 on endosomes to down-regulate MHC-I. (2011). doi:10.1091/mbc.E11-11-0928

36. Viruses, I., Lindwasser, O. W., Chaudhuri, R. \& Bonifacino, J. S. Mechanisms of CD4 Downregulation by the Nef and Vpu Proteins of Primate Mechanisms of CD4 Downregulation by the Nef and Vpu Proteins of Primate Immunodeficiency Viruses. (2007). doi:10.2174/156652407780059177

37. Swigut, T., Shohdy, N. \& Skowronski, J. Mechanism for down-regulation of CD28 by Nef. EMBO J. 20, 1593-1604 (2001).

38. Pawlak, E. N. \& Dikeakos, J. D. Biochimica et Biophysica Acta HIV-1 Nef : a master manipulator of the membrane traf fi cking machinery mediating immune 
evasion MHC-I Downregulation By Nef MHC-I Secretory Pathway Transport PACS-2 MHC-I. BBA - Gen. Subj. 1850, 733-741 (2015).

39. Buffalo, C. Z., Iwamoto, Y., Hurley, J. H. \& Ren, X. crossm. 1-18 (2019).

40. Khan, N., Gowthaman, U., Pahari, S. \& Agrewala, J. N. Manipulation of costimulatory molecules by intracellular pathogens: Veni, Vidi, Vici!! PLoS Pathog. 8, (2012).

41. Singh, R. K., Lau, D., Noviello, C. M., Ghosh, P. \& Guatelli, J. C. An MHC-I cytoplasmic domain/HIV-1 nef fusion protein binds directly to the $\mu$ subunit of the AP-1 endosomal coat complex. PLoS One 4, 1-7 (2009).

42. Subauste, C. S., de Waal Malefyt, R. \& Fuh, F. Role of CD80 (B7.1) and CD86 (B7.2) in the immune response to an intracellular pathogen. J. Immunol. 160, 1831-40 (1998).

43. Köchli, C. et al. CD80 and CD86 costimulatory molecules on circulating T cells of HIV infected individuals. Immunol. Lett. 65, 197-201 (1999).

44. Ahmad, N. NIH Public Access. Life Sci 88, 980-986 (2011).

45. Vanhove, B. Co-stimulatory blockade of the CD28 / CD80-86 / CTLA-4 balance in transplantation : impact on memory T cells ? 6, 1-11 (2015).

46. Wishart, D. NMR Spectroscopy and Protein Structure Determination: Applications to Drug Discovery and Development. Curr. Pharm. Biotechnol. 6, $105-120$ (2005).

47. Konarev, P. V et al. PRIMUS : a Windows PC-based system for small-angle scattering data analysis PRIMUS : a Windows PC-based system for smallangle scattering data analysis. 1277-1282 (2003).

48. Franke, D. \& Svergun, D. I. DAMMIF, a program for rapid ab-initio shape determination in small-angle scattering. 342-346 (2009). 
doi:10.1107/S0021889809000338

49. Volkov, V. V \& Svergun, D. I. small-angle scattering. 860-864 (2003).

50. Kozin, M. B. \& Svergun, D. I. Automated matching of high- and low-resolution structural models research papers Automated matching of high- and lowresolution structural models. 33-41 (2001). 


\section{Main Figures}


Graphical Abstract

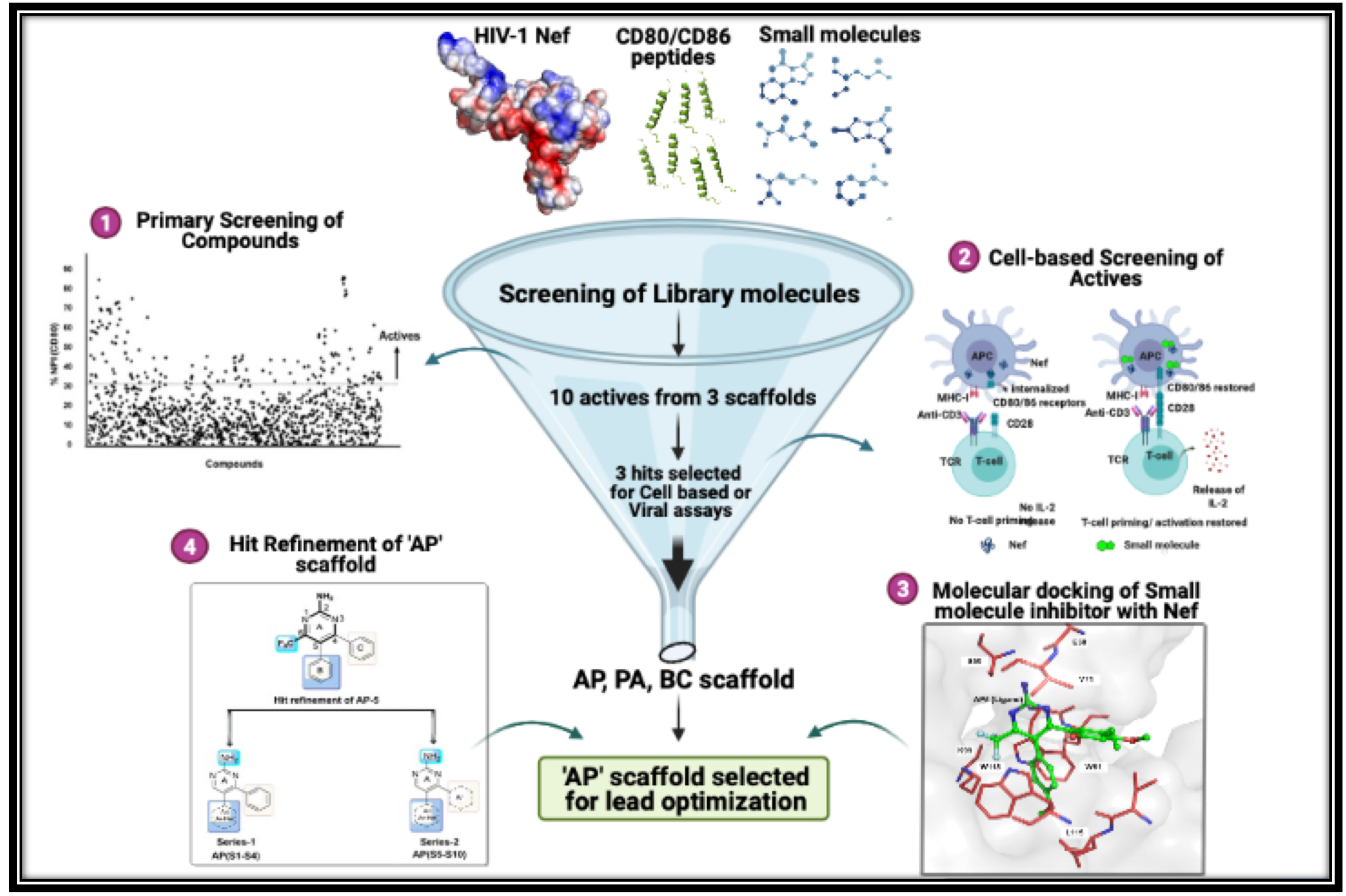


Fig. 2 a

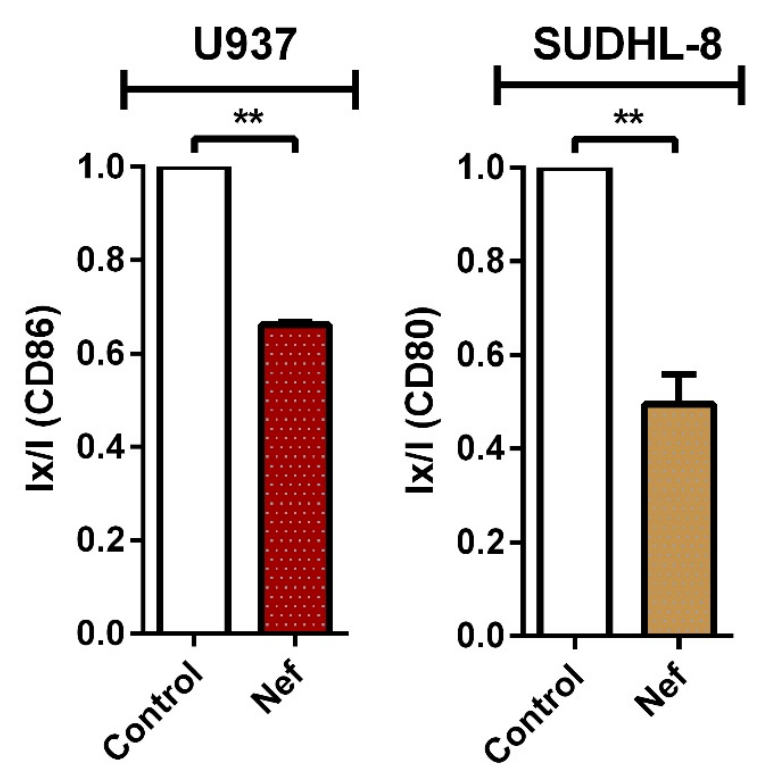

C

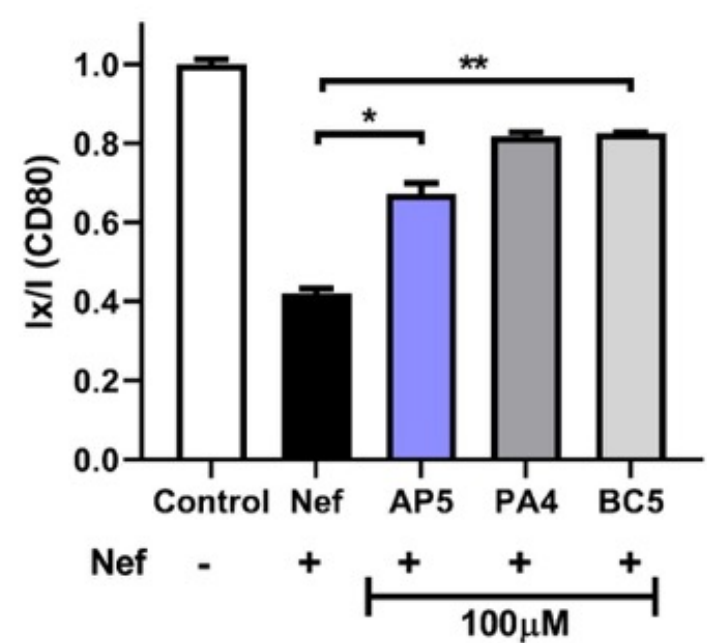

RAJI

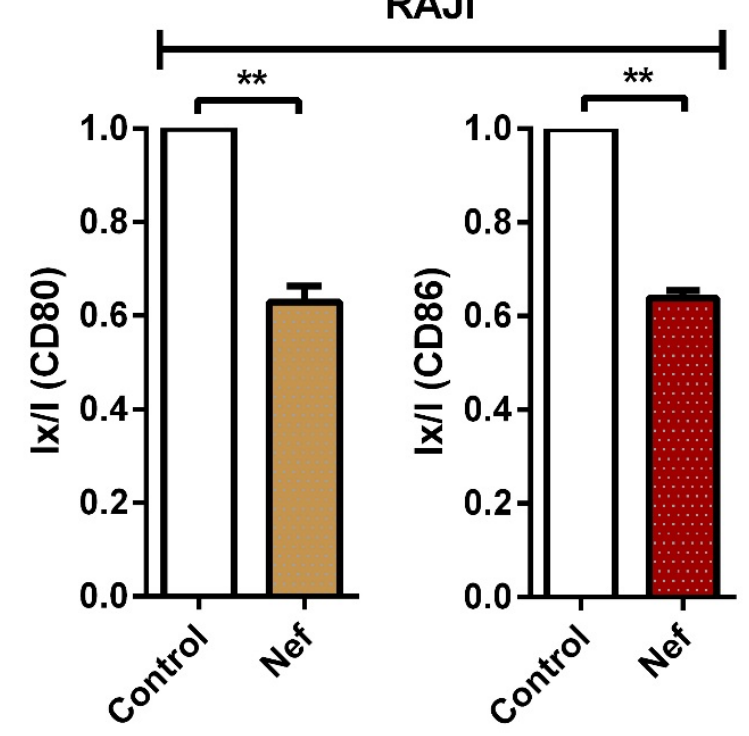

b

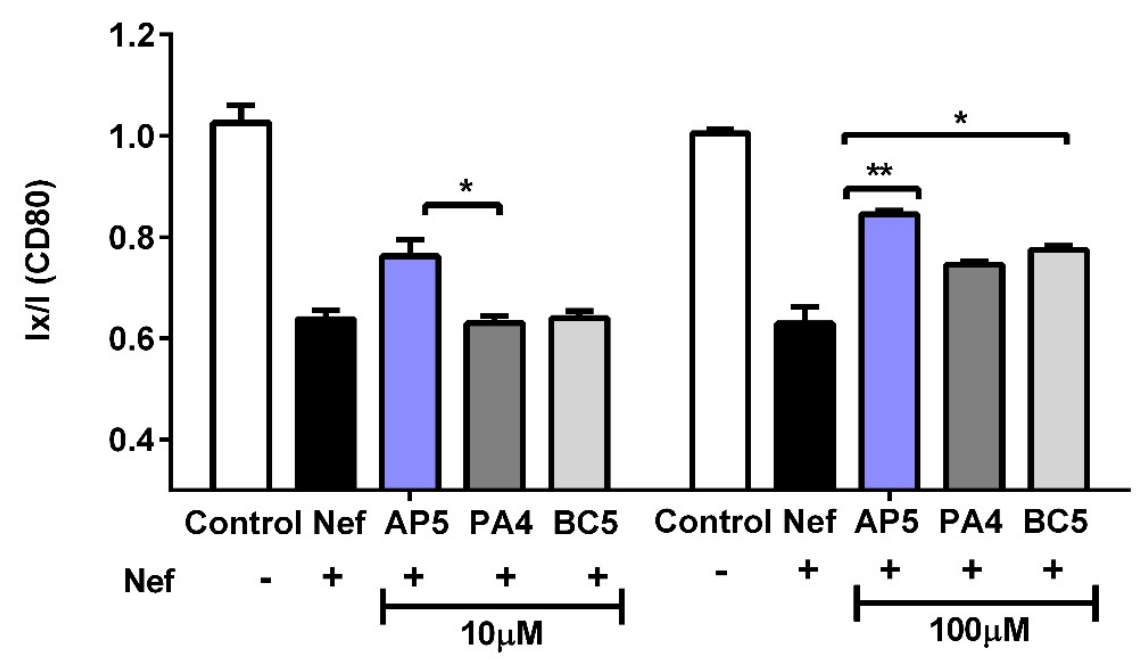

口 YFP Control virus

d

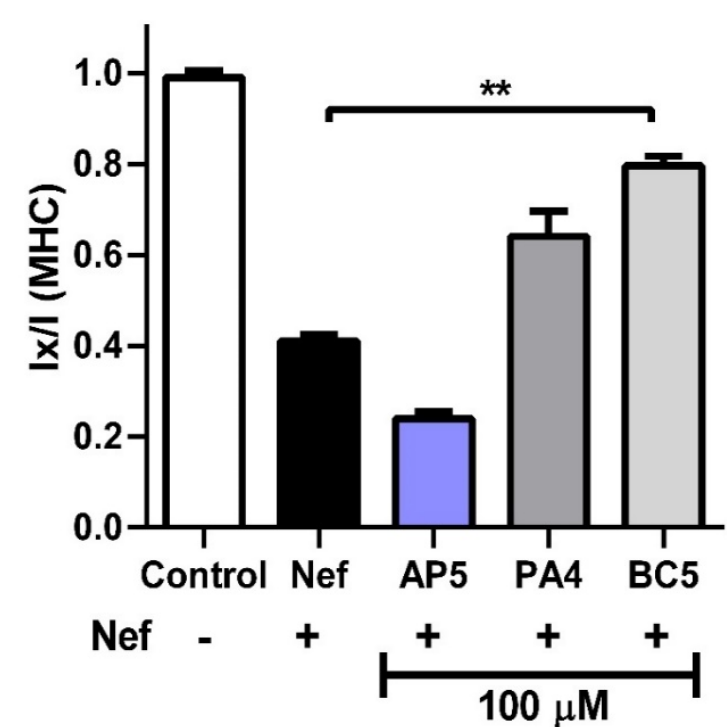

Fig. 2: Cell based assay screening of active compounds from ELISA. (a) Nef mediated down-regulation of surface CD80 or CD86 in 3 different cell lines as indicated. FACS data showing the normalized surface levels of $C D 80 ; I_{x} / l\left(y\right.$ axis) where $I_{x}$ is the average fluorescence intensity in the indicated condition (from a triplicate) and $I$ is the Median of normalized negative control (No Nef control) (b) FACS data shows restoration of CD80 receptors in RAJI cell line after pre-treatment with 3 representative compounds AP5, PA4 and BC5 at 10 and $100 \mu \mathrm{M}$ for $24 \mathrm{~h}$ and analysis after $2 \mathrm{~h}$ post Nef protein delivery (c) RAJl cell line was infected with viral particles (Nef-YFP and YFP alone control cells) in viral infection assay and surface CD80 receptors with compounds were measured by flow cytometry (d) Effects of inhibitors on Nef-MHC-I interactions. RAJI cells were treated with compounds at $100 \mu \mathrm{M}$ and then stained with anti-MHC-I antibody. MHC-I was detected by flow cytometry and shown as Ix/I plots. Compound AP5 shows no restoration of MHC-I indicating its specificity for the Nef-CD80 interface. 


\section{Fig. 3}

a

C

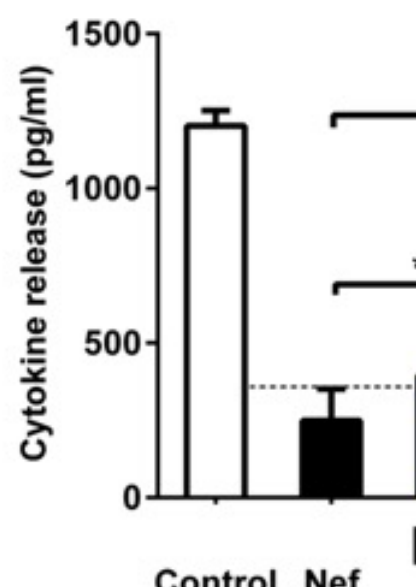

Virus $^{\text {nef+ }}$

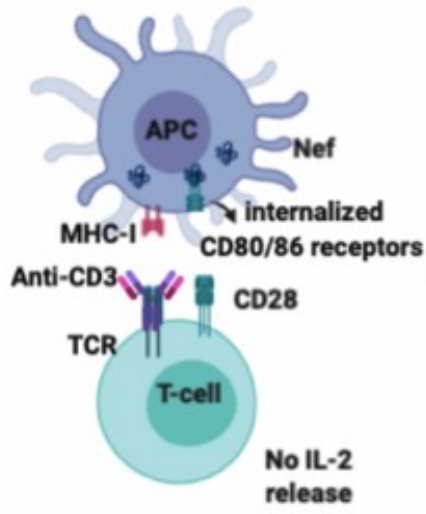

No T-cell priming

if Nef

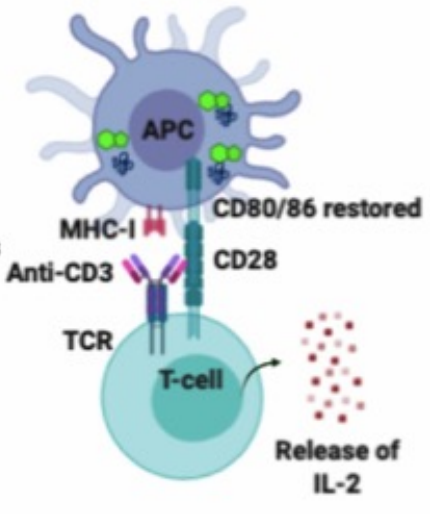

T-cell priming/ activation restored

(o) Small molecule b

T-cells: B cells (with anti-CD3 Ab)

T-cells: B cells (no anti-CD3 Ab)

T cells + anti-CD3 Ab

T cells alone-

$\mathrm{B}$ cells + anti-CD3 Ab-

$B$ cells alone-

0

Cytokine release (pg/ml)

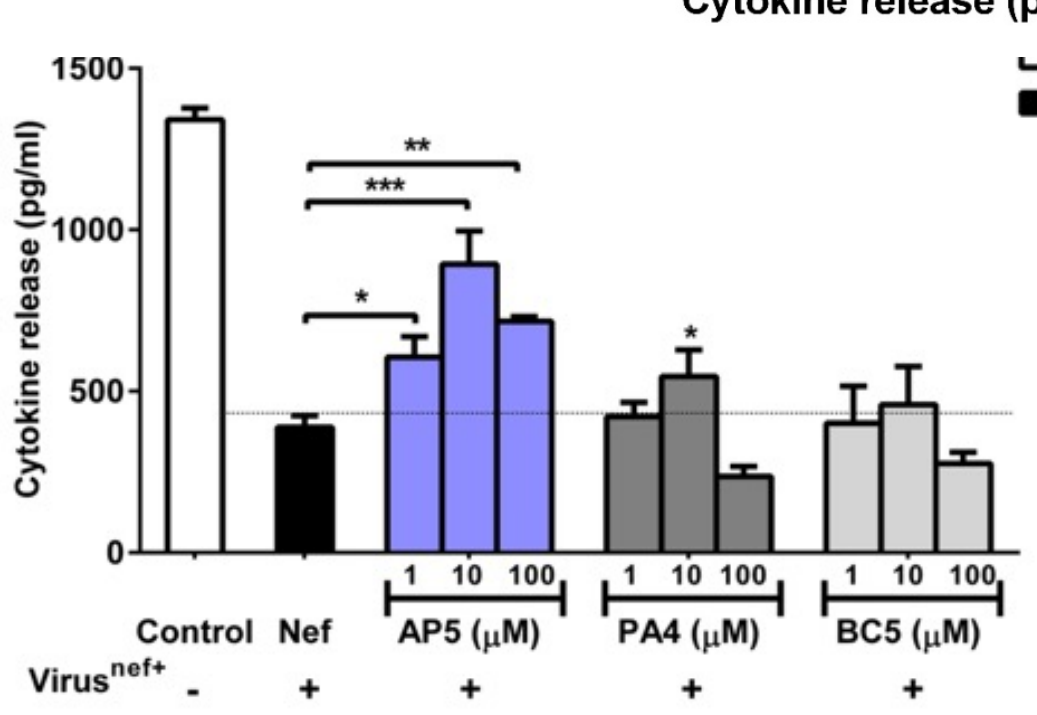

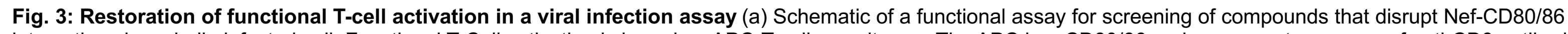

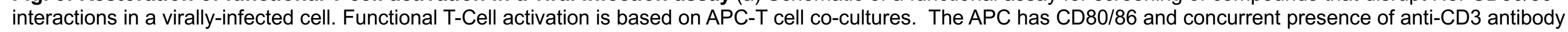

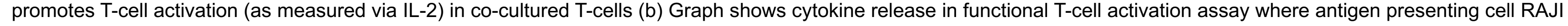

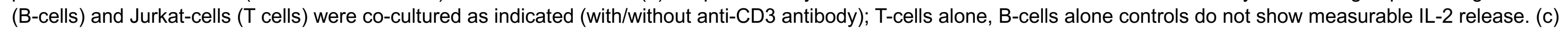

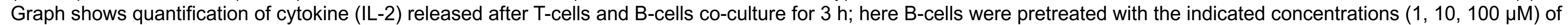

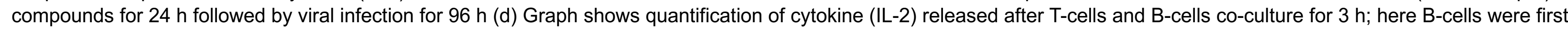

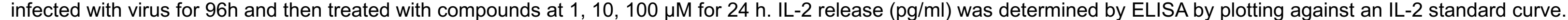
Note: viral infection reduces IL-2 release, and all 3 compounds showed a dose dependent restoration of IL-2 release. AP5 showed IL-2 release at $1 \mu$ M. 


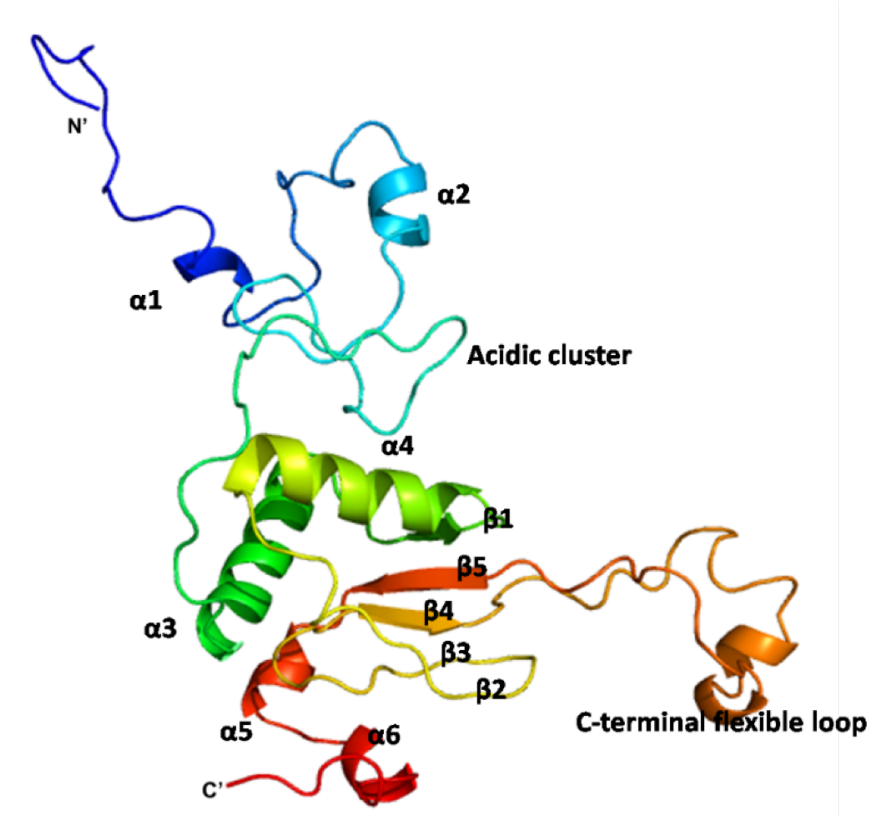

C
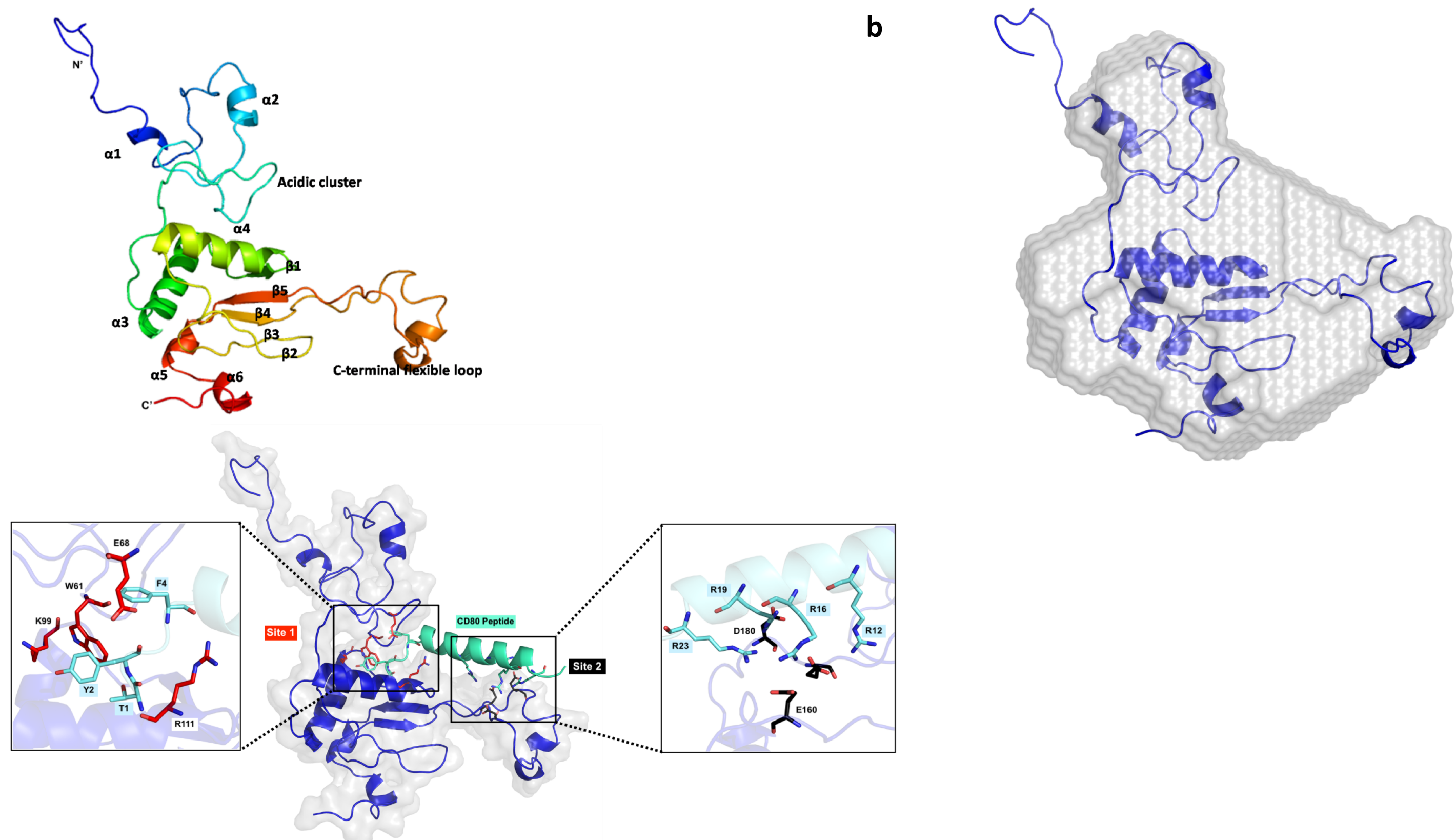

Fig. 4: Modelling of Nef with multi-template computational approach (a) Cartoon representation of the predicted structure of Nef shows flexible N-terminal region and well-conserved core domain, colored in accordance with their position ( $\mathrm{N}$-terminal in blue to $\mathrm{C}$-terminal in red) with respective $\alpha$-helices and $\beta$-sheets as indicated. (b) The ab initio shape of the solution structure of the Nef (blue color) from the SAXS data (grey surface) fits well with the computational model (cartoon representation) (c) Surface representation of HIV-1 Nef is depicted with the best CD80 binding pose. CD80 peptide in a docked pose (cyan color) in Nef obtained using SiteMap program. The inset shows the important residues of Nef involved in interaction with CD80 at Sites 1 and 2. 
Fig. $5 \quad a$

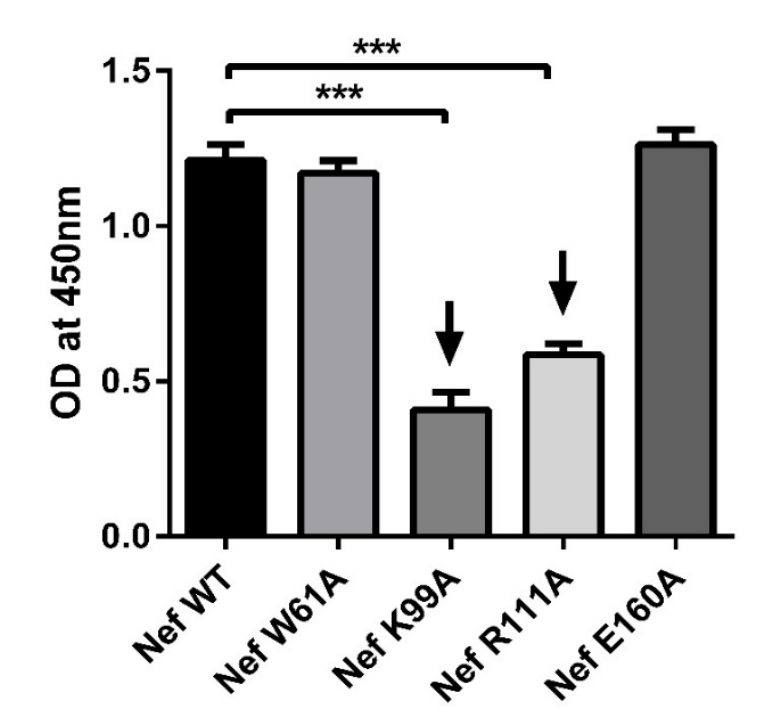

C

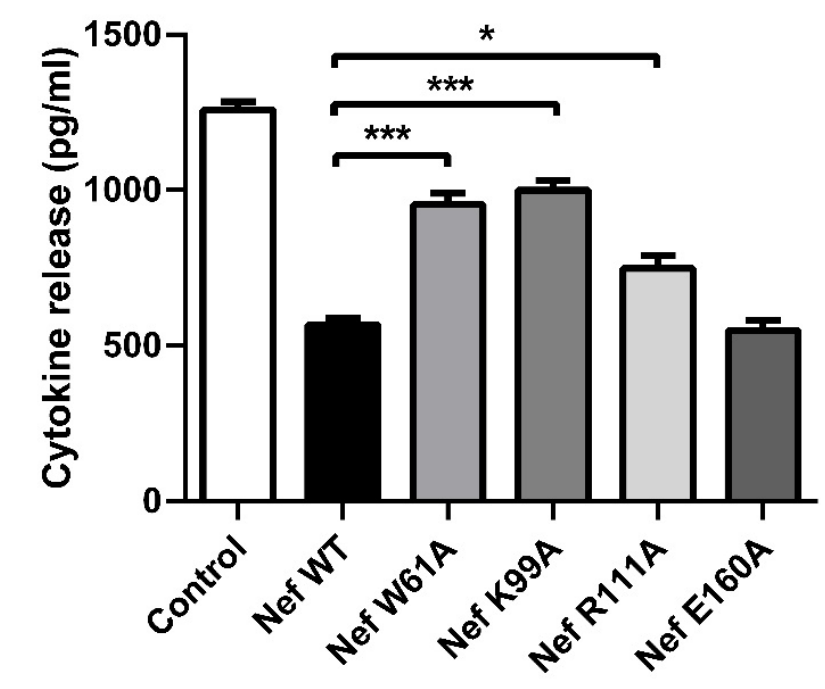

b

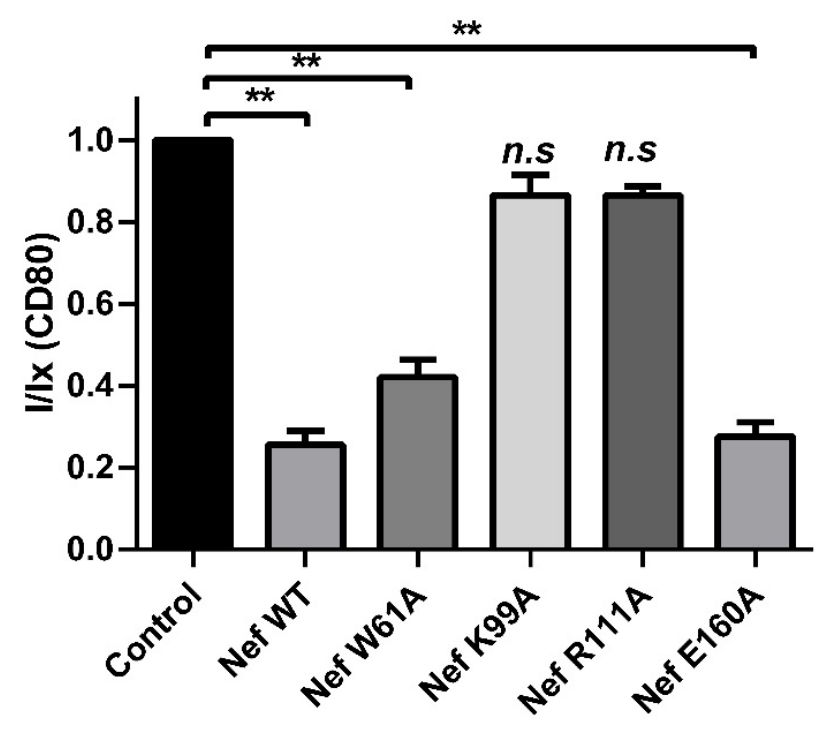

d

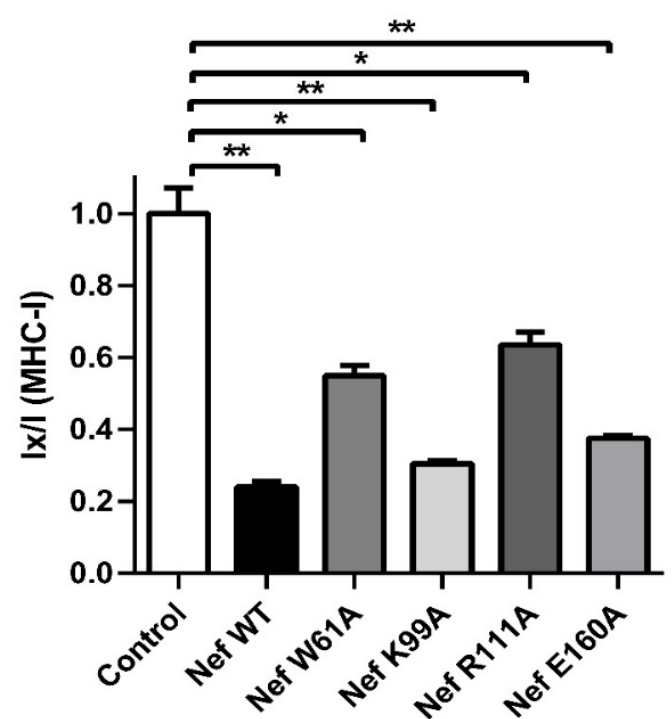

Fig. 5: Evaluation of interaction between CD80 and Nef mutants in biochemical and cell-based assays (a) Graph shows colorimetric signal of immobilized CD80 cytosolic peptide upon binding to Nef ${ }^{W T}$ or Nef mutants as measured by ELISA at OD450nm. Two mutants Nef ${ }^{\mathrm{K} 99 \mathrm{~A}}$ and Nef ${ }^{\mathrm{R} 111 \mathrm{~A}}$ showed reduced affinity to CD80 peptide (b) Graph shows FACS data of surface levels of CD80 receptors in RAJI cell line after delivery of Nef ${ }^{\mathrm{WT}}$ or Nef mutant protein delivery . No significant down regulation seen with mutants Nef ${ }^{\mathrm{K} 99 \mathrm{~A}}$ and Nef ${ }^{\mathrm{R} 111 \mathrm{~A}}$ (c) Graph shows the levels cytokine (IL-2) released in supernatants of cells in the co-culture functional T-cell activation assay after delivery of the Nef mutants as compared to the wild type Nef protein. (d) Graph shows FACS data of MHC-1 levels after delivery with Nef ${ }^{\mathrm{WT}}$ and mutants. Nef ${ }^{\mathrm{WT}}$ or mutants were delivered into RAJI cells using Chariot ${ }^{\mathrm{TM}}$ delivery reagent. MHC-I was detected by flow cytometry and shown as Ix/I plots. 
Fig. 6

a

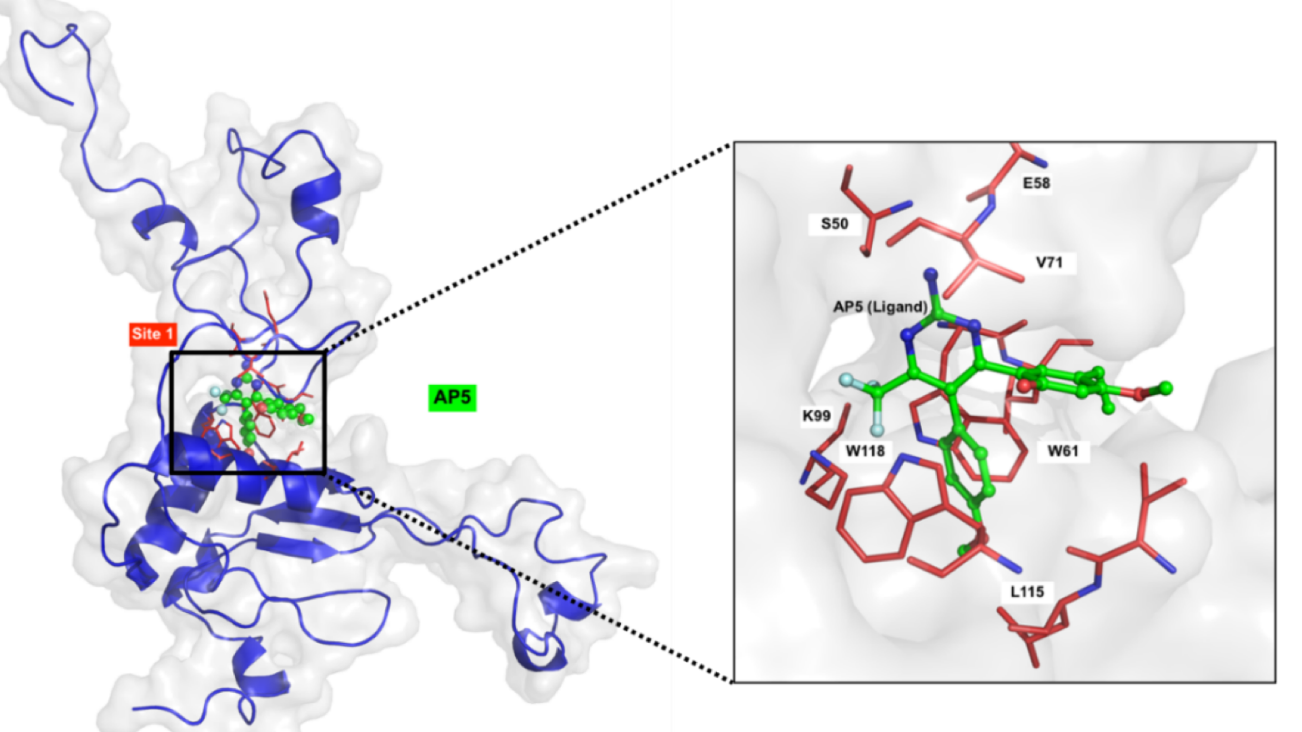

C

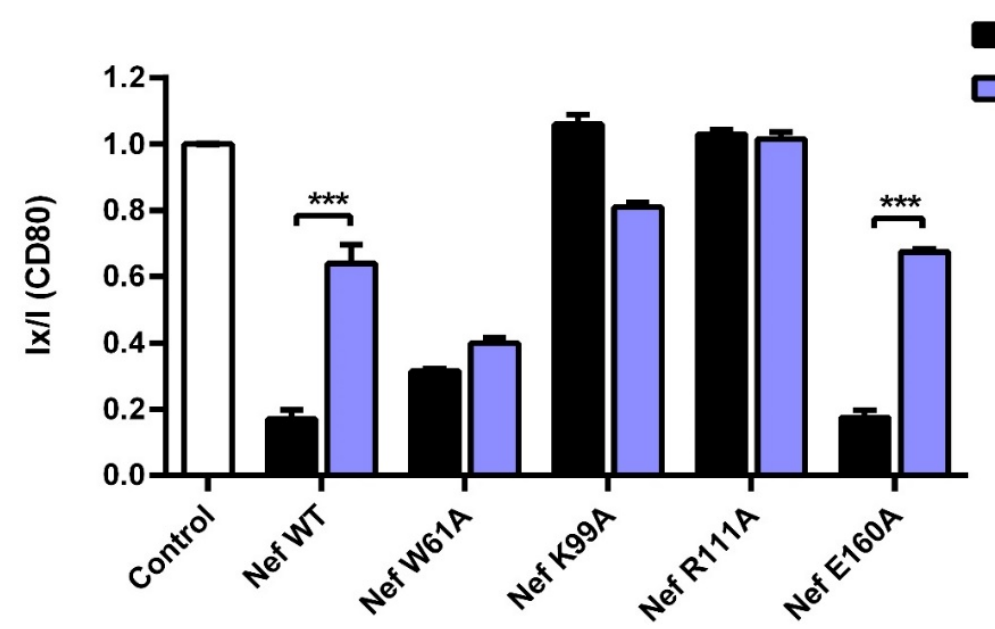

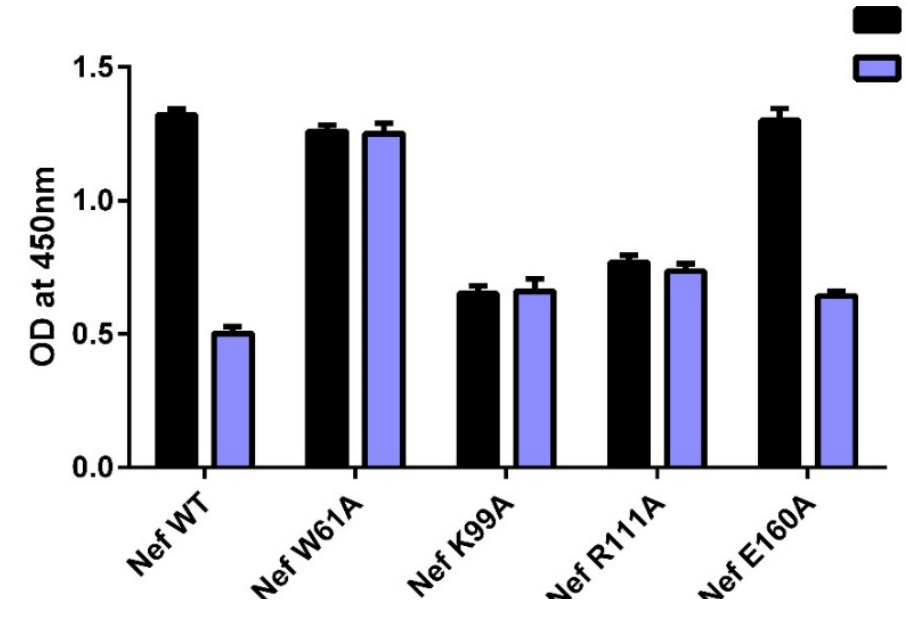

Nef variants

Nef +10 $\mu$ M AP5

d

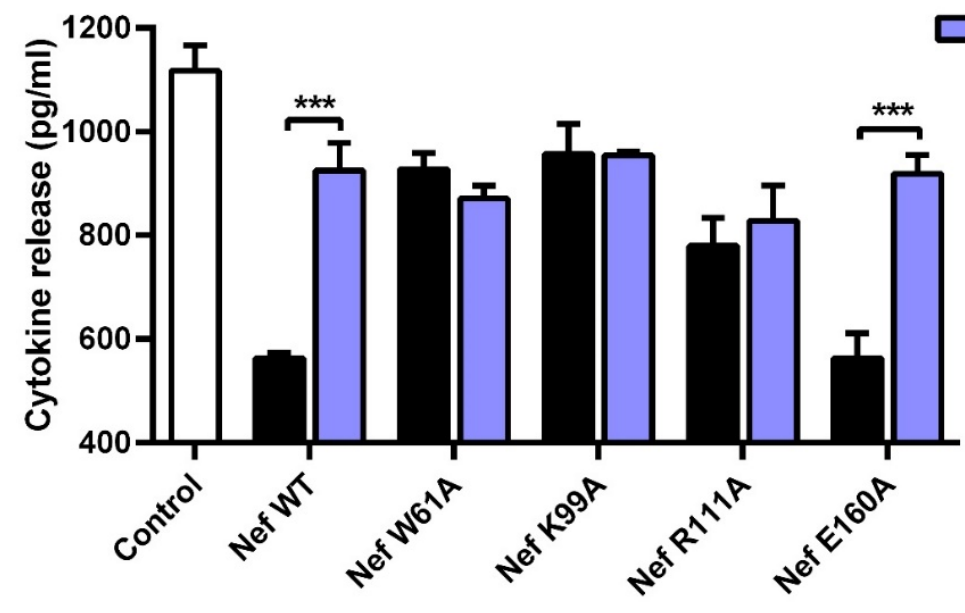

Fig. 6: Structural and functional evaluation of the interaction between Nef and AP5 (a) Surface representation of HIV-1 Nef depicting AP5 ligand (green color) binding. The binding site of AP5 molecule overlaps with the CD80 binding site (Site-1). The inset shows the important residues for the interaction between AP5 and Nef. The non-polar residues such as $\mathrm{W}_{61}, \mathrm{~L}_{91}, \mathrm{I}_{109}$ and $\mathrm{L}_{115}$ contribute to hydrophobic interactions with $\mathrm{CF}_{3}$. AP5 ligand docking studies shows that the binding interactions occurs between the $\alpha 4$ and $\alpha 5$ helices along with few residues such as $\mathrm{W}_{61}$ $\mathrm{E}_{65}$ and $\mathrm{R}_{111}$ which are crucial for AP5-Nef interaction (b) Graph shows colorimetric signal of immobilized CD80 cytosolic peptide upon binding to NefWT or Nef mutants in the presence labsence of $10 \mu \mathrm{M}$ AP5 as measured by ELISA at OD450 nm. (c) Graph shows surface levels of CD80 receptors in RAJl cell line after the delivery of NefWT or Nef mutant protein delivery as measured by FACS in the presence /absence of $10 \mu \mathrm{M}$ AP5. NefW61A, NefK99A and NefR111 did not show any further change in CD80 levels with AP5 addition. (d) Graph shows cytokine (IL-2) release in supernatants after the co-culture T-cell activation assay. RAJl cells were pre-treated with $10 \mu \mathrm{M}$ AP5 for $1 \mathrm{~h}$ and then the cells were delivered with Nef mutants or wild type Nef protein for $2 \mathrm{~h}$ before co-culture with Jurkat T-cells for $3 \mathrm{~h}$. The IL-2 levels remain unchanged with and without addition of AP5 compound in all three mutants Nef(W61A, Nefk99A and NefR111. Reduction in IL-2 seen with mutant NefE160A comparable to NefWT. 
Fig. 7

a

C

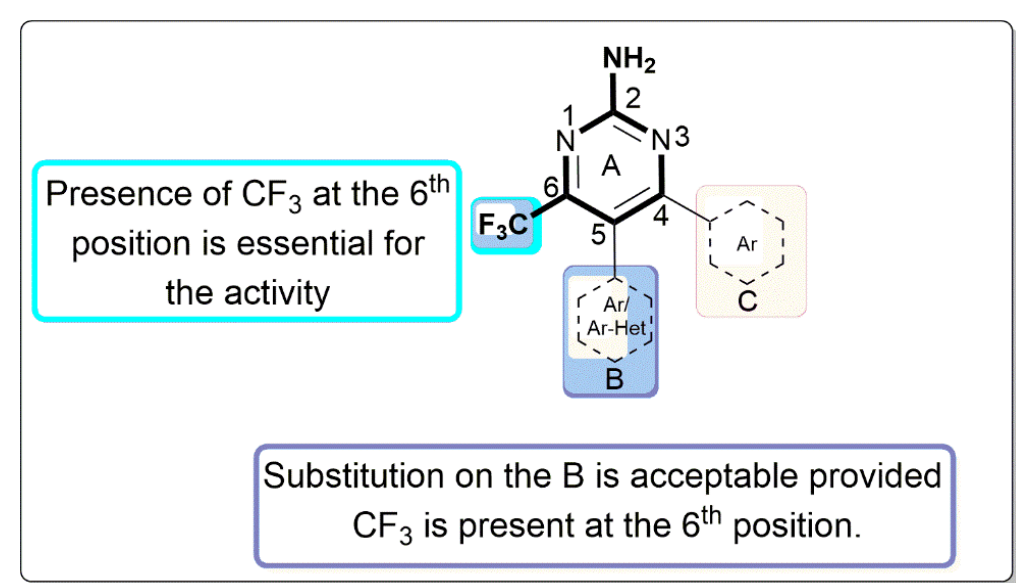

b

\begin{tabular}{|c|c|c|c|c|c|c|}
\hline & & & & & 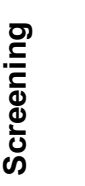 & 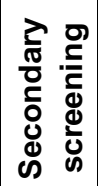 \\
\hline $\begin{array}{r}\text { Sample } \\
\text { Codes }\end{array}$ & $-\xi-\mathrm{CF}_{3}$ & $\xi \bar{B}$ & $-\xi-\bar{c}\rangle$ & \begin{tabular}{|c|} 
CD80 \\
NPI
\end{tabular} & $\begin{array}{c}\text { CD86 } \\
\text { NPI }\end{array}$ & $\begin{array}{c}\text { Cell } \\
\text { based } \\
\text { assay }\end{array}$ \\
\hline AP-S1 & - & $3 \|$ & & & & \\
\hline AP-S2 & - & $-\xi-\backslash$ & & & & \\
\hline AP-S3 & - & $-\xi-1$ & & & & \\
\hline AP-S4 & - & & & & & \\
\hline AP-S5 & - & & & & & \\
\hline AP-S6 & - & & & & & \\
\hline AP-S7 & - & & & & & \\
\hline AP-S8 & - & & & & & \\
\hline AP-S9 & - & & & & & \\
\hline $\begin{array}{l}\text { AP- } \\
\text { S10 }\end{array}$ & - & & & & & \\
\hline $\begin{array}{l}\text { AP- } \\
\text { S11 }\end{array}$ & $-\left\{-\mathrm{CF}_{3}\right.$ & & & & & \\
\hline
\end{tabular}

$\underline{\mathrm{NPI}}$

Active

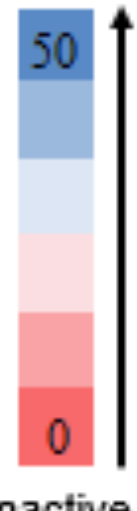

Fig. 7: SAR and Hit refinement with AP5 as a template (a) Scheme shows Medicinal chemistry approach for hit refinement of AP5 showing two series of compounds (b) Summary of SAR strategy to design compounds similar to AP5 structure with modifications made on rings A, B and C as indicated. The synthesized molecules were evaluated for their effect on Nef-CD80/CD86 inhibition (c) Heat map table showing SAR with synthesized compounds. The various substitutions in rings A, B and C are indicated as well as their activity in the ELISA and cell-based assays 